\title{
A MODULE SYSTEM FOR ISOLATING UNTRUSTED SOFTWARE EXTENSIONS
}

\author{
A Thesis \\ Submitted to the Faculty of Graduate Studies and Research \\ In Partial Fulfillment of the Requirements \\ for the Degree of \\ Master of Science \\ in Computer Science \\ University of Regina
}

by

Simon A. Orr

Regina, Saskatchewan

November, 2006

Copyright 2006: S.A. Orr 


$\begin{array}{ll}\begin{array}{l}\text { Library and } \\ \text { Archives Canada }\end{array} & \begin{array}{l}\text { Bibliothèque et } \\ \text { Archives Canada }\end{array} \\ \begin{array}{l}\text { Published Heritage } \\ \text { Branch }\end{array} & \begin{array}{l}\text { Direction du } \\ \text { Patrimoine de l'édition }\end{array} \\ \begin{array}{l}\text { 395 Wellington Street } \\ \text { Ottawa ON K1A ON4 }\end{array} & \begin{array}{l}\text { 395, rue Wellington } \\ \text { Ottawa ON K1A ON4 } \\ \text { Canada }\end{array}\end{array}$

Your file Votre référence ISBN: 978-0-494-29160-3 Our file Notre référence ISBN: $978-0-494-29160-3$

NOTICE:

The author has granted a nonexclusive license allowing Library and Archives Canada to reproduce, publish, archive, preserve, conserve, communicate to the public by telecommunication or on the Internet, loan, distribute and sell theses worldwide, for commercial or noncommercial purposes, in microform, paper, electronic and/or any other formats.

The author retains copyright ownership and moral rights in this thesis. Neither the thesis nor substantial extracts from it may be printed or otherwise reproduced without the author's permission.
AVIS:

L'auteur a accordé une licence non exclusive permettant à la Bibliothèque et Archives Canada de reproduire, publier, archiver, sauvegarder, conserver, transmettre au public par télécommunication ou par l'Internet, prêter, distribuer et vendre des thèses partout dans le monde, à des fins commerciales ou autres, sur support microforme, papier, électronique et/ou autres formats.

L'auteur conserve la propriété du droit d'auteur et des droits moraux qui protège cette thèse. $\mathrm{Ni}$ la thèse ni des extraits substantiels de celle-ci ne doivent être imprimés ou autrement reproduits sans son autorisation.
In compliance with the Canadian

Privacy Act some supporting forms may have been removed from this thesis.

While these forms may be included in the document page count, their removal does not represent any loss of content from the thesis.
Conformément à la loi canadienne sur la protection de la vie privée, quelques formulaires secondaires ont été enlevés de cette thèse.

Bien que ces formulaires aient inclus dans la pagination, il n'y aura aucun contenu manquant.

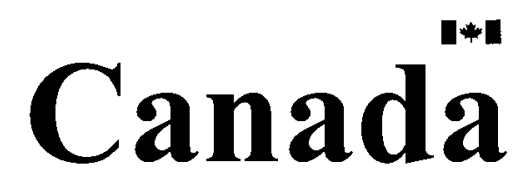




\section{UNIVERSITY OF REGINA}

\section{FACULTY OF GRADUATE STUDIES AND RESEARCH}

\section{SUPERVISORY AND EXAMINING COMMITTEE}

Simon A. Orr, candidate for the degree of Master of Science, has presented a thesis titled, A Module System for Isolating Untrusted Software Extensions, in an oral examination held on September 13, 2006. The following committee members have found the thesis acceptable in form and content, and that the candidate demonstrated satisfactory knowledge of the subject material.

External Examiner: $\quad$ Dr. Mohamed El-Darieby, Faculty of Engineering

Supervisor: $\quad$ Dr. Philip Fong, Department of Computer Science

Committee Member: $\quad$ Dr. Howard J. Hamilton, Department of Computer Science

Committee Member: $\quad$ Dr. Malek Mouhoub, Department of Computer Science

Chair of Defense: $\quad$ Dr. Martin Argerami, Department of Mathematics and Statistics 


\begin{abstract}
With the recent advent of dynamically extensible software systems, in which software extensions may be dynamically loaded into the address space of a core application to augment its capability, there is a growing interest in protection mechanisms that can isolate untrusted software components from a host application. Existing languagebased environments such as the Java Virtual Machine (JVM) and the Common Language Infrastructure (CLI) achieve software isolation by an interposition mechanism known as stack inspection. Expressive as it is, stack inspection is known to lack declarative characterization and is brittle in the face of evolving software configurations.

In this thesis, a run-time module system, called IsoMoD, is proposed for the Java platform to facilitate software isolation. With ISOMOD, a core application may create namespaces dynamically and impose arbitrary name visibility policies to control whether a name is visible, to whom it is visible, and in what way the name can be accessed. Because ISOMOD exercises name visibility control at load time, loaded code runs at full speed. Furthermore, because IsoMoD's access control policies are maintained separately, they can evolve independently from core application code. In addition, the ISOMOD policy language provides a declarative means for expressing a very general form of visibility constraints. Not only can the IsoMOD policy language simulate a sizable subset of the permissions available in the Java 2 security architecture, it does so with policies that are robust to changes in software configurations. The IsOMoD policy language is also expressive enough to completely encode a capability type system known as Discretionary Capability Confinement. In spite of its expressiveness, the IsoMOD policy language admits an efficient implementation strategy. In short, IsoMoD avoids the technical difficulties of interposition by trading off an acceptable level of expressiveness. Therefore, name visibility control in the style of IsoMoD is a lightweight alternative for language-based access control.
\end{abstract}




\section{Acknowledgments}

I would like to express my appreciation to Dr. Philip Fong, my supervisor, for providing me with a sequential learning program and financial support, and for helping me conduct the research outlined in this program. He worked hard supervising and training me to become a good writer and researcher. His kindness and strictness will help me in future research.

I am grateful to the Faculty of Graduate Studies and Research and the Department of Computer Science for their financial assistance which allowed me to concentrate on my research.

This work is supported in part by a Discovery Grant from NSERC.

I would also like to thank the other members of my supervisory committee, Dr. Malek Mouhoub and Dr. Howard Hamilton, for their valuable comments and suggestions on my thesis. 


\section{Dedication}

I dedicate this work to my parents, Andrew and Marianne Orr, who support me in all my endeavors. 


\section{Contents}

Abstract $\quad$ i

Acknowledgments $\quad$ ii

Dedication $\quad$ iii

Table of contents $\quad$ iv

List of figures vii

1 INTRODUCTION 1

1.1 Motivation ....................... 1

1.2 Contributions ....................... 2

1.3 Thesis organization .................. 4

2 LANGUAGE BASED ACCESS CONTROL 6

2.1 Interposition . . . . . . . . . . . . . . 6

2.1 .1 Stack inspection . . . . . . . . . . . . . . 6

2.1 .2 Inlined reference monitor . . . . . . . . . . . . 9

2.1 .3 Assessment . . . . . . . . . . . . . . 10

2.2 Namespace management . . . . . . . . . . . . . . . . 11

2.2 .1 Safe-Tcl . . . . . . . . . . . . . . . . 12

2.2 .2 Scheme $48 \ldots \ldots \ldots \ldots$

2.2 .3 SPIN ............................ 14

2.2 .4 Assessment . . . . . . . . . . . . . . 15

2.3 Encapsulation . . . . . . . . . . . . . . . 15

2.3.1 Encapsulation as visibility control . . . . . . . . . 15

2.3.2 Encapsulation policies . . . . . . . . . . . . 17 


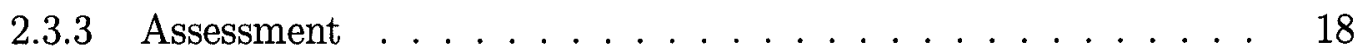

3 IsoMod $\quad 19$

3.1 The IsoMod security architecture . . . . . . . . . . . 19

3.1.1 Delegation-Style class loading . . . . . . . . . . . . . 19

3.1 .2 The IsoMod Approach . . . . . . . . . . . . . 20

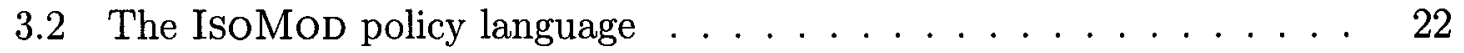

4 SAMPLE APPLICATIONS

4.1 Selective hiding of system services . . . . . . . . . . . 25

4.2 Systematic control of reference acquisition . . . . . . . . . . . 27

4.3 Discretionary Capability Confinement . . . . . . . . . . . . . . 30

5 IMPLEMENTATION EXPERIENCE 35

5.1 Design rationales . . . . . . . . . . . . . . 35

5.2 Type mirrors . . . . . . . . . . . . . . . . . 37

5.2 .1 Structure of mirrors . . . . . . . . . . . . 38

5.2.2 Staged construction of mirrors . . . . . . . . . . . 40

5.3 Staged verification . . . . . . . . . . . . . . . 42

5.4 Policy evaluation .................... . . 45

5.5 Summary .............................. 47

6 PERFORMANCE EVALUATION 49

6.1 Introduction . . . . . . . . . . . . . . . . 49

6.2 Methodology ......................... 50

6.3 Tractability .......................... 54

6.4 Composition of overhead . . . . . . . . . . . . 55

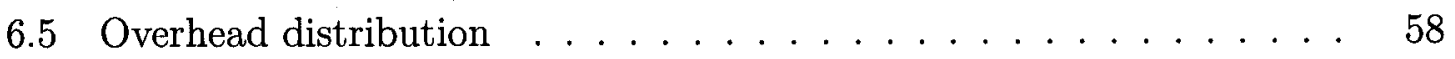

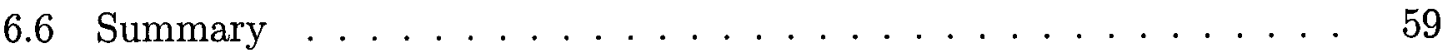


7 CONCLUSION $\quad 61$

7.1 Related work . . . . . . . . . . . . . . . . 61

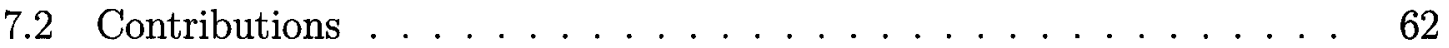

7.3 Limitations and future work . . . . . . . . . . . . . 64

A AN IsoMod POLICY FOR DCC 66

B EXPERIMENTAL DATA $\quad 70$

List of References $\quad 71$ 


\section{List of Figures}

2.1 Monitoring via Interposition . . . . . . . . . . . . 7

2.2 Stack Inspection . . . . . . . . . . . . . . . . . . 7

2.3 Stack Inspection with Amplified Rights . . . . . . . . . . . . 8

2.4 IRM Rewriter . . . . . . . . . . . . . . . . 9

2.5 Safe-Tcl Security Mechanism . . . . . . . . . . . . . . . 12

3.1 Hierarchical Namespaces and IsoMod . . . . . . . . . . . . 20

3.2 Access Rights . . . . . . . . . . . . . . . . . . 22

3.3 Built-in Predicates on Declared Types . . . . . . . . . . . . . . 22

3.4 Built-in Functions on Declared Types, Methods, and Fields . . . . . . 23

3.5 Operational Semantics for Authorization . . . . . . . . . . . . 24

4.1 DCC Type Constraints . . . . . . . . . . . . . . . . . . . 31

5.1 Preloading Algorithm . . . . . . . . . . . . . . . 38

5.2 Preloading Example . . . . . . . . . . . . . . . 43

6.1 Benchmarking Suite . . . . . . . . . . . . . . 52

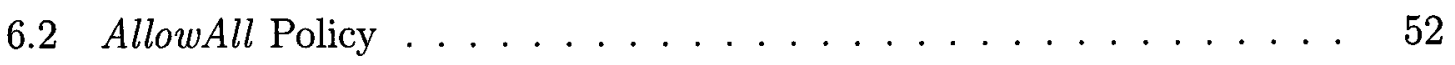

6.3 Java Access Control $(J A C)$ Policy . . . . . . . . . . . . . . . 53

6.4 IsoMon Overhead . . . . . . . . . . . . . . . 55

6.5 Composition of Overhead . . . . . . . . . . . . 56

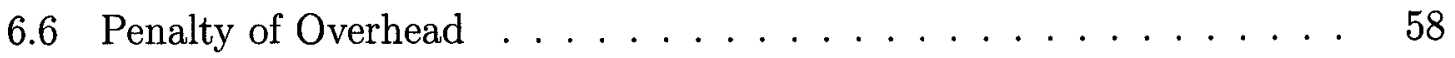




\section{INTRODUCTION}

\subsection{Motivation}

In modern day computing, a successful software system must anticipate the evolution of software requirements. This gives rise to a family of software systems known as dynamically extensible systems, in which the functionality of a core application can be augmented dynamically by loading, linking, and invoking program code units that were not originally part of the core application. Dynamically extensible systems find their uses in mobile code platforms, scriptable applications, and systems with the plug-in architectures. In each case, trusted and untrusted code units are loaded and executed in the same run-time environment. The challenge of secure cooperation [28] is to protect these mutually suspicious code units from one another while they are executing within the same run-time environment.

An established paradigm for addressing the challenge of secure cooperation is language-based security [32]. Specifically, untrusted code units are encoded in a safe language, and subsequently executed in a secure run-time environment, the protection mechanisms of which are implemented by programming language technologies such as type systems, program rewriting and execution monitoring.

Most existing language-based approaches to access control are based on the classical notion of interposition [1,36, 37, 39]. A direct implementation of this idea is to interpose monitoring code at the entry points of security relevant system services. At run time, authorization decisions are made by examining invocation arguments or execution history. In the Java Virtual Machine (JVM) [21] and the Common Language Infrastructure (CLI) [9], a direct implementation of this approach is found. Specifically, stack inspection [37], the main access control mechanism of the JVM and the CLI, is essentially a form of interposition. Direct interposition, however, is difficult to maintain. Security checks are scattered over the entire host system. Fixing 
a vulnerability requires the availability of host system source code. Worst still, as security checks are hard-coded into the host system, evolution in security requirements or software configuration is not easily addressed without reprogramming the host system itself.

A second language-based approach to implement interposition is by load-time binary rewriting $[10,36,37,39]$ Specifically, monitoring code is weaved into untrusted code at load time. Although this so called Inlined Reference Monitor (IRM) approach [36] is equal in expressive power to direct interposition [16], the former has clear software engineering advantages over the latter. In particular, the late binding of security checks allows security code to evolve separately from the rest of the system, thereby addressing the software engineering concerns raised in the previous paragraph. Unfortunately, independent reports have confirmed that the injected code incurs significant run-time overhead $[36,37]$. For example, in [36], up to $70 \%$ slow down was observed if domain-specific optimization was not introduced.

There is an obvious engineering dilemma in the design of interposition-based access control mechanisms. By embedding security checks in the host system, direct interposition fails to support the evolution of security requirements and software configurations in a graceful manner. Maintaining the security policy offline, IRM has the advantage of anticipating software evolution, but it incurs significant runtime overhead.

\subsection{Contributions}

The research question investigated by this thesis is as follows. Is interposition (direct or IRM-based) always necessary for access control in the context of dynamically extensible systems? Interposition is motivated by the need for execution monitoring [31], in which the dynamic state and the execution history of a system are examined 
when authorization decisions are made. In many cases, one simply wants to completely turn off a system service. (This is evident in the large number of target-less BasicPermissions defined in the Java 2 security architecture [15].) In other cases, the safety property [31] to be enforced is memory-less, and the avoidance of the confused deputy problem [17] is not a significant concern. In such contexts, execution monitoring can be replaced by a lighter-weight enforcement mechanism that does not exhibit the engineering dilemma presented by interposition.

This thesis examines a seldom studied point in the design space of language-based access control, specifically, name visibility control. The intuition is that, if the name of the entry point for a system service is not visible to an untrusted code unit, then the service is essentially inaccessible to the code unit. Therefore, access control can be achieved by specifying what names are visible, to whom they are visible, and to what extent they are visible. These aspects of name visibility are specified by the scoping rules of a programming language. Such rules are usually fixed for modern block-structured programming languages. In order to use name visibility control as an access control mechanism, two ingredients are necessary: (1) a policy language for specifying custom scoping rules that constrain the visibility of names, and (2) a protection mechanism that allows an application to impose custom scoping rules over a local namespace in which untrusted code resides. In the programming language literature, a facility that is responsible for managing the visibility of names across namespaces [18] is called a module system.

The goal of this research is to investigate the degree to which name visibility control can serve the purpose of access control when full-fledged execution monitoring is not necessary. To this end, a module system, IsoMoD, is proposed as a practical security architecture for dynamically extensible Java applications. Because IsoMOD exercises name visibility control only at load time, and does not inject any monitoring code into classfiles, loaded code runs at full speed. Furthermore, because IsoMoD 
access control policies are maintained separately, they evolve independently from core application code.

An intriguing finding of this study is that a rich family of access control policies can be expressed as name visibility constraints. The IsOMOD policy language provides a declarative means for expressing a very general form of visibility constraints. Not only can the IsoMoD policy language simulate a sizable subset of permissions in the Java 2 security architecture, it can do so with policies that are robust to changes in software configurations. The IsoMoD policy language is also expressive enough to completely encode a capability type system known as Discretionary Capability Confinement [12]. In spite of its expressiveness, the IsoMoD policy language admits an efficient implementation strategy.

In short, ISOMOD avoids the technical difficulties of interposition by trading off an acceptable level of expressiveness. Therefore, name visibility control in the style of IsOMOD is a lightweight alternative to interposition for language-based access control.

\subsection{Thesis organization}

The remainder of this thesis is organized as follows.

Chapter 2 provides a survey of past works in language-based access control. Two main approaches to language-based access control are surveyed: (1) interposition and (2) name visibility control. An assessment of each approach, especially its impact on security, is also provided. The discussion is rounded off by relating name visibility control to the classical notion of encapsulation.

Chapter 3 provides an overview of the IsoMoD security architecture and policy language. The architecture of IsoMoD is introduced as a refinement of the delegation model of class loading. How it can be used by an application core to isolate untrusted 
software extensions is described. An informal account of the syntax of the IsoMoD policy language is provided. The operational semantics of the policy language is specified in the form of an authorization algorithm.

Chapter 4 presents some sample applications of IsoMoD to illustrate the utility of its policy language. Three example applications are given. The first example demonstrates how to selectively hide system services. The second example illustrates how to systematically control the acquisition of resource references, and to do so in a way that accommodates evolving software configurations. Finally, the IsoMoD policy language is employed to completely encode a capability type system, thereby demonstrating the expressiveness of the policy language.

Chapter 5 discusses an implementation strategy for IsoMoD. The technical challenges addressed by this implementation strategy are first described. A three-staged verification scheme is then presented, including the data structure it maintains, and the algorithm by which verification steps are carried out. Every verification step involves the evaluation of IsoMoD policy clauses. The method for policy evaluation is also covered in this chapter.

Chapter 6 presents the experiments and analysis that were conducted in evaluating the performance of IsoMoD. The experimental methodology is first presented. A first experiment quantifies the overhead of IsoMoD. The result of this experiment is analyzed to reveal how various components of the verification process contribute to the overhead. A second experiment examines the distribution of IsoMoD's overhead in a longer executing application.

Chapter 7 concludes the thesis with a discussion of contributions, limitations, and future work. 


\section{LANGUAGE BASED ACCESS CONTROL}

This chapter provides a survey of classical works in language-based access control. Language-based security, such as typed assembly language [23] and proof-carrying code [24], which deal mainly with memory safety, will not be covered. We begin our survey in Section 2.1, by examining the notion of inserting monitoring code at security relevant program points. Section 2.2 highlights some of the work done in the area of namespace management. We conclude our survey with a discussion in Section 2.3 , which deals with encapsulation as a visibility control mechanism.

\subsection{Interposition}

In the past, language-based access control was achieved mainly through interposition, where monitoring code was inserted at security relevant program points. Calls made to these program points are checked against an access control policy which either denies or allows the call to proceed (Figure 2.1). Two classical variants of this approach discussed in this section are, stack inspection and Inlined Reference Monitors.

\subsubsection{Stack inspection}

Stack inspection's [37] main purpose is to allow components with diverse origins to share the same runtime environment, and access its resources in a controlled manner, according to their respective levels of trust. Stack inspection is a history-based access control protection mechanism. Method invocations cause frames to be placed on a run-time stack (Figure 2.2). Principals representing privileges are associated with the declaring classes of the methods, and thus every frame is associated with a set of rights. These rights determine what operations a method can and cannot perform. Methods invoking methods will cause a build up of frames to appear on the run-time stack (Figure 2.2). 


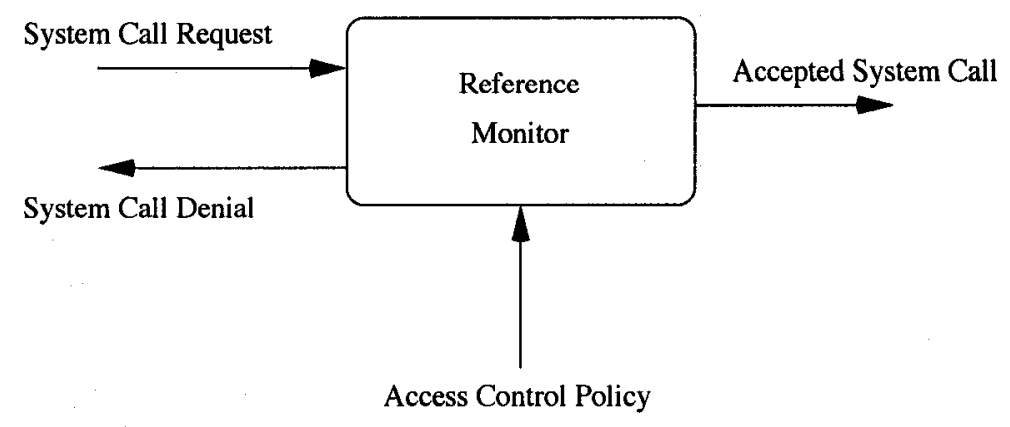

Figure 2.1: Monitoring via Interposition

\begin{tabular}{|l|l|}
\hline Method 4 & $\{$ P1 $\}$ \\
\hline Method 3 & $\{$ P1, P4 $\}$ \\
\hline Method 2 & $\{$ P1, P3, P4 $\}$ \\
\hline Method 1 & $\{$ P1, P2, P3, P4 $\}$ \\
\hline
\end{tabular}

Figure 2.2: Stack Inspection

Security relevant methods are interposed with monitoring code. When one of these methods is invoked, the stack inspection algorithm [37] commences. The algorithm inspects each frame appearing on the run-time stack from newest to oldest. Each frame is inspected further to determine its set of rights. If a frame is found to be lacking the rights required by the operation, the stack inspection algorithm denies the requested method invocation. Thus, the set of rights granted to the top-most frame, is the intersection of all rights appearing on the run-time stack.

This method of stack inspection as implemented in the JVM [21] and CLI [9], solves two non-trivial problems, namely the confused deputy problem [17] and rights amplification. These problems drive the remaining discussion on stack inspection.

Confused Deputy This problem occurs when an untrusted code unit employs the help of some trusted code unit (the Deputy) to perform a sensitive operation. The untrusted code unit lacks the required privileges, while the Deputy posses them. Unaware that the call originated from an untrusted source, the Deputy performs the requested operation, and commits a security violation. 


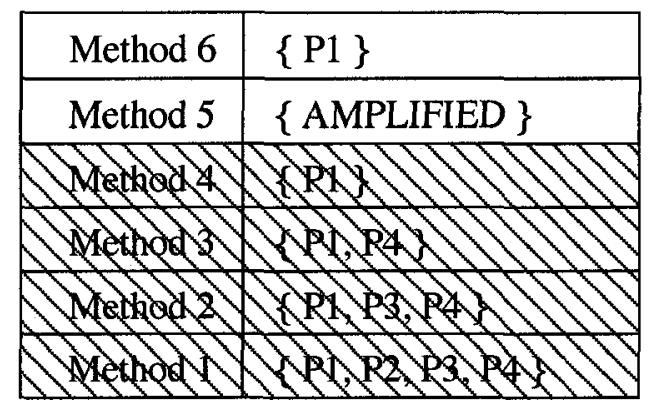

Figure 2.3: Stack Inspection with Amplified Rights

Untrusted frames on the run-time stack have fewer privileges associated with them, while trusted frames have more privileges. When a sensitive operation is invoked, the stack inspection algorithm [37] described above commences. Through this examination of a program's execution history, the algorithm determines that the invocation of the security relevant method originated from an untrusted source, thus denying its invocation. By determining the originating invoker, the confused deputy problem can be solved.

Rights Amplification As described above, calls originating from an untrusted source will not be allowed to invoke security relevant system services. In some cases, a system service may be required to perform a privileged operation. This operation could consist of the system service writing to a "log" file for debugging purposes. Since the rights granted to a frame is the intersection of all rights appearing on the run-time stack, a system service appearing at the top of the run-time stack may lack the necessary privileges to perform a sensitive operation. To compensate for this lack of privileges, the system service can "amplify" its own rights by modifying its stack frame to include the amplified principal. This enhanced privilege acts as a stopping barrier in the stack inspection checking algorithm. When the amplified rights principal is encountered, no further checks are done beyond that frame in the stack. An example of this is demonstrated in Figure 2.3. The grayed out portion is not checked by the stack inspection algorithm, because the previous frame contains 


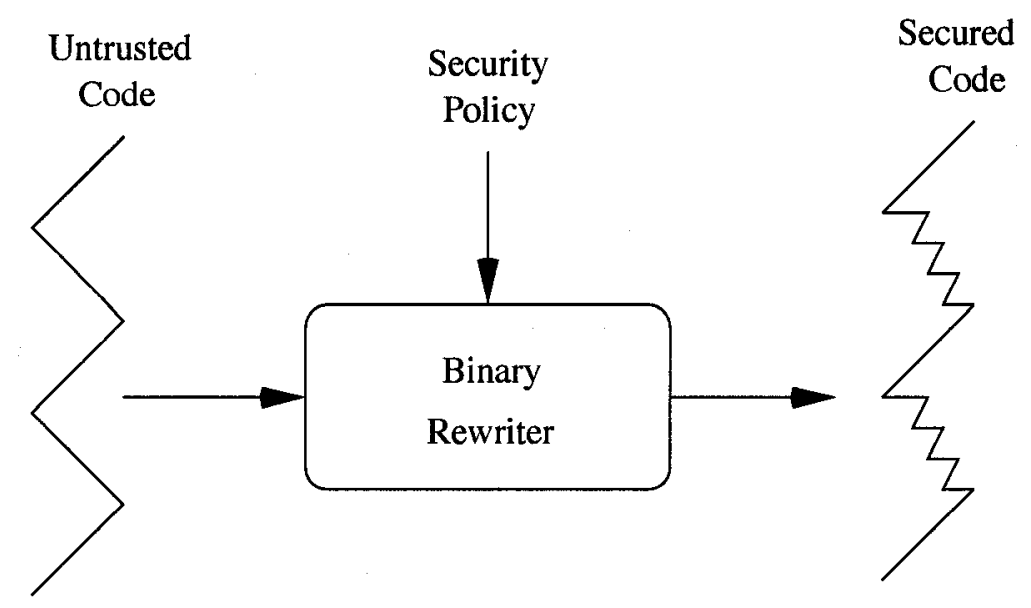

Figure 2.4: IRM Rewriter

the amplified rights principal. This method of amplifying allows system services to perform privileged operations when necessary.

While stack inspection is efficient at performing security checks, its design does not allow for easy evolution of its security architecture. Because monitoring code is embedded directly in the source, a complete audit is necessary in order to apply "fixes". While this approach is possible, it is impractical in large scale systems.

\subsubsection{Inlined reference monitor}

Inlined Reference Monitors [35, 36] are another incarnation of interposition, and were designed partly to address the weaknesses of embedded security checks and their inability to evolve efficiently. Policy enforcing code exists outside the program, and is weaved into untrusted code by a trusted rewriter, making the code unit secure (see Figure 2.4). During this rewrite, guards are inserted at security relevant program points, such as system services. When a request is made of a system service, the guard checks the caller and its arguments against an access control policy to either deny or allow the operation.

Inlined Reference Monitors offer the advantageous feature of policies being able to evolve independently of the program code. While this particular feature solves a 
problem inherent to stack inspection, it suffers a drawback to its design. The guards which perform the access control checks do so at run time, thus resulting in poor run-time performance. This fact is demonstrated in the following works.

In a classical work [36], Inlined Reference Monitors were used to simulate Java's stack inspection. In this implementation, additional information was passed around to the guards which replicated the information in the JVM's run-time call stack. Through the passing of this information, guards examined the information to determine if the invocation of a call originated from an untrusted source. It was through this examination that the same power of stack inspection's ability to perform access control was achieved.

In a second independent work [37], another IRM-like implementation of stack inspection, known as security passing style, was developed. In this implementation, methods were rewritten to pass around a new variable which contained security information. This variable was inspected by the guards in order to make security decisions.

While these approaches allowed for the graceful evolution of an application, two independent experiments confirmed that the run-time overhead incurred from Inlined Reference Monitors is much larger $[36,37]$ than an embedded approach like JVM's stack inspection.

\subsubsection{Assessment}

Monitors inserted at security relevant program points effectively guard the services from illegal invocation. Stack inspection and Inlined Reference Monitors are able to solve the confused deputy problem and rights amplification, but each suffer from drawbacks to their implementations.

In stack inspection, security checks are embedded directly in the source. A vulnerability to the security architecture requires access to the source, as well as a complete 
audit in order to apply a fix. Worse still is that as a security policy evolves, a complete reprogramming of the system source is required.

Inlined Reference Monitors solved the problem of security requirement evolution by maintaining the security policies independent of the system source and were demonstrated to be equal in power to interposition [16]. Since Inlined Reference Monitors weave monitoring code directly into the program, the run-time overhead incurred by these checks is significant as reported by two independent studies [36, 37].

\section{$2.2 \quad$ Namespace management}

Another approach to language-based access control is through namespace management. A namespace, in the programming language sense, specifies the denotation of symbolic names for some program region. It provides a mapping from symbolic names to the code units they represent. If the mapping has an association for a given symbolic name, then that name is visible in the program region. The process by which the run-time environment looks up the code unit represented by a symbolic name is called name resolution. Namespace management is the technique of controlling the visibility of names and their resolution to code units in a namespace. By controlling how a name is resolved, access control to code units can be achieved. A name can be removed (hidden) from a namespace, in effect turning the code unit (service) "off" (i.e., name visibility control). If a service is required, but its use could be potentially dangerous, a name can be linked to a code unit which employs further checks on the caller's execution history and its arguments, against some access control policy before delivering the service (i.e., name resolution control).

A trusted code unit is allowed to use system services with minimal security restrictions. These trusted code units are placed in a namespace with few access control restrictions imposed upon them. Untrusted code units are placed in a separate names- 


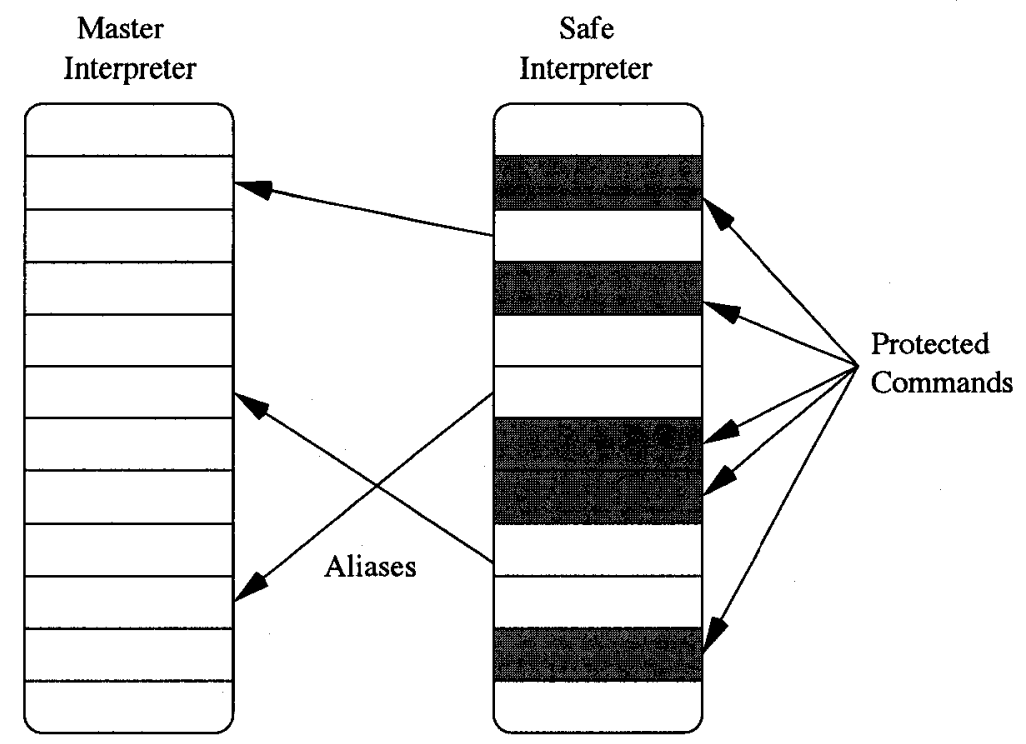

Figure 2.5: Safe-Tcl Security Mechanism

pace, where potentially dangerous services are disabled (name visibility control), or the name of the service is resolved to a code unit that performs further checks (name resolution control). This technique of namespace management is used extensively to enforce language-based access control in early works such as Safe-Tcl, Scheme 48, and SPIN. Their implementations are discussed in the following sections.

\subsubsection{Safe-Tcl}

Safe-Tcl [25] employs name visibility and name resolution control to allow scripts written in Tcl to execute safely. Distinct binding environments are setup to host trusted and untrusted scripts. An environment is a namespace, in that it maps names to scripts. Associated with each environment is an interpreter responsible for reading and executing the scripts.

A master interpreter is responsible for executing trusted scripts. These scripts have minimal restrictions and are free to interact with the system in ways that would be dangerous for untrusted scripts. Each untrusted script is placed in a padded cell (Figure 2.5). These padded cells are composed of safe interpreters that can only 
execute safe commands. Potentially dangerous commands are protected by hiding their names in the environment through name visibility control (Figure 2.5). When an untrusted script requires the use of a system service (as many useful scripts do), precautions are taken to ensure the command is used in a safe manner. The names of unsafe commands are interpreted through aliases.

An alias is an association between a command in the safe interpreter and a command in the master interpreter. The master interpreter makes available a set of potentially dangerous commands through this aliasing. An alias causes further checks to occur in the master interpreter on the caller and its arguments (name resolution control). By this process, it is easy to protect potentially dangerous commands from being invoked. Also, if the need arises, it is easy to redirect the call to some code unit to perform extra checks to ensure particular security.

\subsubsection{Scheme 48}

Scheme 48 ensures that cooperation between independent agents (users or subsystems) will not endanger the resources of value to an agent [28]. An agent resides in and is evaluated through its own binding environment. In order for an agent to access a resource, the name of the resource must be resolved in the environment. This resolution creates a link from the name to the resource being accessed. Agents within the system are granted privileges through their ability to reference objects by name.

In order to protect an agent's resource, an environment is constructed for that agent where names for the resource are either visible or hidden. If a name exists, then the resource can be accessed. If the name does not exist, the resource is inaccessible. This creation of binding environments for agents is a form of name visibility control.

In the case of cooperation, agents may share objects between themselves. This sharing could be potentially dangerous, as one agent may wish to view data that another agent deems private. In this sense, the program of the source agent (the 
one supplying the resource) passes the resource to a third-party and indicates which actions are allowed to be taken on the resource. The target agent (the one wishing to use the resource) supplies its program (sequence of operations to be performed on the resource) to the third-party as well. The third-party validates the target agent's program against the actions which are allowed to be performed and some security policy. The result of the program is then returned to the target agent. This control over security sensitive resources is a form of name resolution control.

\subsubsection{SPIN}

SPIN [4] is an operating system which allows extensions to be written to extend the capability of the system while protecting the core services. Some services in SPIN are made extensible by providing interfaces to their operations. If an operation is not part of an interface, then it cannot be used by extension code, and thus it cannot be extended.

The extensions are written in a type-safe language, namely Modula-3. The reason for this choice is to enforce security properties at the language level, and let other concerns, such as type-safety be handled by the language.

Extensions, when loaded, exist within what are known as logical protection domains. These protection domains are namespaces that contain code and exported interfaces representing views on system resources. The interfaces can be modified to allow different extensions to posses different views on the available system resources. In order to protect security relevant resources from potentially dangerous use, their operations are omitted from their respective interfaces (name visibility control). While this effectively protects system resources, it does not allow for complete extensibility.

In order to provide complete extensibility, further steps are taken on security relevant resources. These resources must be interacted with through extensions reg- 
istering handlers. After a handler is registered, it can receive notification of events. Events are generated when a protected resource is accessed by an application, which directs the event to the registered handler. Before an extension can register a handler however, the primary handler is contacted with the name of the module and the event it wishes to be informed about. The primary handler determines if the extension is allowed to handle the event or not. This process is analogous to setting up guards on potentially dangerous events. These guards can be expressed in terms of predicates which perform simple applicability evaluations. This type of guarding events is a form of name resolution control. With this approach, a system can provide extensibility, and also ensure safe use of its protected operations.

\subsubsection{Assessment}

Namespace management consists of two aspects. Names can either be turned "on" or "off" (name visibility control) or names can be linked to wrapper code which performs additional checks (name resolution control).

In some cases it may be undesirable to completely turn "off" a service, as this can be too restrictive. Instead we would prefer to present a restricted "view" of that service so that it can only be used in a certain way by a particular party.

Name resolution control is really a form of IRM in which weaving is performed by a linker. Seen in this way, it suffers from the same performance drawback of IRM that was highlighted previously.

\subsection{Encapsulation}

\subsubsection{Encapsulation as visibility control}

In the programming language literature, encapsulation is a standard technique for supporting the graceful evolution of modules [26]. A module is composed of its 
exported interface and its implementation details. The interface specifies the set of operations that can be performed on the module, while the implementation describes the actions of each operation.

In a classic work by Snyder [33], encapsulation is defined as:

... a technique for minimizing interdependencies among separately-written modules by defining strict external interfaces. The external interface of a module serves as a contract between the module and its clients, and thus between the designer of a module and other designers.

A modules interface is visible to all clients, while the implementation is hidden from them, allowing the implementation to evolve without necessitating changes to client code that depends on the interface.

Since a client of a module must use the module interface to interact with the module, the module interface can be used to enforce access control. Since the interface exports a list of operations that can be applied to an object, this list can be modified to reduce the possible operations allowed. In an extreme example, if all operations are removed from a module's interface, then no operations can be performed on the object.

In modern object-oriented programming languages, such as Java, multiple interfaces to a class instance are defined with the access modifiers public, private, and protected. Each modifier denotes a particular category of clients to which the class member is visible.

public This member is visible to all clients

protected Clients existing in the same package or a subclass of the restricting class can view this member

private The member is only visible to its declaring class 
This separation of clients into categories provides a form of name visibility control. The three categories can be thought of in the following terms: public induces global visibility, protected induces conditionally visibility, and private induces local visibility. While this approach to access control is effective in restricting the visibility of class members, it can be too inflexible at times. The access modifiers are tightlycoupled with the class during its construction, and there are only a fixed, limited number of client categories. The work on encapsulation policies [30] addresses this very issue.

\subsubsection{Encapsulation policies}

Encapsulation policies were designed to address the weaknesses of object-oriented languages. It was discovered that access rights are inseparable from classes, client categories are fixed, and access rights not customizable [30]. The main reason for these weaknesses is the tight coupling between the class and the rights given at construction time. In order to solve these particular problems, it was necessary to separate the encapsulation policies from the classes [30].

A class defining an encapsulation policy is defining an external interface. Multiple policies are constructed to present different views of a class. This technique defines multiple usage contracts, while keeping the original implementation of the class. An example policy may be one that presents an unrestricted view of all the class's members (all). Another policy may only present members of a class that allow for queries to be performed on the class's internal data (get). A client using a class, chooses which policy to apply to that class. A client may choose to only query a class's data by choosing the get policy, or the client may choose the less restrictive policy with all.

It is in this sense that while visibility control is presented with multiple views of a class, it does not enforce security. Giving the client the right to choose which 
members of a class it wants can easily be seen as an insecure approach to access control.

\subsubsection{Assessment}

Encapsulation policies effectively separate the interface and implementation of a class. A class that defines multiple policies can present clients with different views. While this approach can achieve visibility control, it does not enforce security. A client chooses which policy it wishes to apply to the class, and in doing so, chooses in which way to use the class. A client is not obligated to choose the most restrictive policy to serve its purpose.

In order for a class to present multiple views of itself, multiple policies must be defined, such as all and get. While it is easy to define multiple policies for a class, the process soon becomes infeasible when the number of classes is large. Also, in order to define a new visibility restriction to be placed on all classes, a new policy must be defined for each of the applicable classes. Again, in a system with a large number of classes, this becomes impractical. It is these weaknesses in the design of encapsulation policies that IsoMoD addresses with its design. 


\section{IsoMod}

This chapter provides a overview of IsOMOD, describing both its architecture (Section 3.1) and its policy language (Section 3.2).

\subsection{The IsoMod security architecture}

IsoMoD employs name visibility control as the sole mechanism for access control. The following describes the Java class loading mechanism from the perspective of name visibility control.

\subsubsection{Delegation-Style class loading}

In programming language terminology, a Java class loader is the mirror [5] of a runtime namespace. An instance of the ClassLoader class is employed by the JVM to load the object code of classes, and to define the classes in the namespace mirrored by the ClassLoader instance. The object code of a class is transported through a network or stored in a file system in an intermediate representation known as a classfle. Class definition [21, Section 5.3.5] is the process by which a classfile is converted into a class object in the JVM. Programmers may define a custom subclass of the ClassLoader class, thereby overriding the procedure by which the JVM locates classfiles (e.g., loading classfiles from the World Wide Web), or introducing a classfile preprocessing step prior to class definition (e.g., injecting instrumentation code). Once a class $C$ is defined by a class loader $L, L$ is said to be the defining class loader [20] of $C$.

Hierarchical organization of namespaces is enabled by the delegation model of class loading [20], in which the names visible in a parent namespace is imported implicitly into a child namespace (Figure 3.1). Specifically, the set of class names visible in a namespace $L$ is the union of (1) the set of class names visible in the parent of $L$ plus (2) the set of class names that are defined locally by $L$. How is this effect achieved 


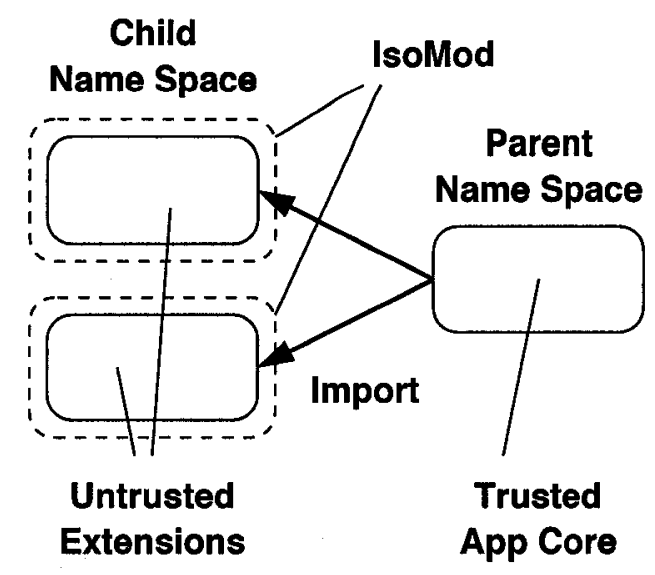

Figure 3.1: Hierarchical Namespaces and IsoMoD

algorithmically? Associated with every class loader $L$ is another class loader, called the delegation parent of $L$. The class loaders thus form a delegation hierarchy, at the root of which is the bootstrap class loader. To look up the Class object of a given class name in a class loader $L$, the delegation parent of $L$ is first consulted. If a Class object of the specified name is defined by the delegation parent, then that Class is returned. Otherwise, the delegation parent of that delegation parent is consulted, and so on. When none of the delegation ancestors of $L$ defines a Class of the given name, then $L$ will load and define that class (if it has not already done so). This class is then returned as the result of class look up.

A class may refer to external entities such as other classes or their fields and methods. These external references are resolved in the same namespace in which the referring class is defined (i.e., via the defining class loader of the referring class). In this way, static scoping is enforced.

\subsubsection{The IsoMod Approach}

In a dynamically extensible software system, the trusted application core is defined in a parent namespace, while child namespaces are created for defining untrusted software extensions (Figure 3.1). Core application services are exposed to the exten- 
sion code by implicitly importing names from the core application namespace to the extension namespace. ISOMOD is a run-time module system designed for isolating untrusted software extensions. It does so by controlling the visibility of names in the namespaces in which untrusted software extensions reside. Specifically, an IsoMoD namespace enforces two kinds of control: (1) restricting the visibility of names that are imported from the parent namespace, and (2) restricting the visibility of locally defined names. When a name is placed under visibility control, an IsoMoD namespace may (a) control which locally defined class can "see" the name, and (b) present an alternative, restricted view of the entity to which the name is bound. Every IsoMOD name space is endowed with a custom name visibility policy, which specifies visibility restrictions to be imposed on the names visible in the namespace. When appropriately constructed, an IsOMOD policy may be used to selectively hide core application services from untrusted extensions (Section 4.1 and 4.2), or impose collaboration protocols among classes defined in the extension namespace (Section 4.3). A major contribution of this work is the design of a policy language that can express a rich family of access control policies as fine-grained visibility constraints.

An ISOMOD namespace is an instance of a user-defined class loader class. An IsoMoD class loader performs extra checks on a classfile before converting it into a Class object. Specifically, class definition is only authorized when no external accesses in the classfile are denied by the policy. This late enforcement (i.e., load time) of visibility control distinguishes IsoMoD from traditional module systems, in which visibility control is enforced only at compile time. It is this feature that makes the IsOMOD module system into a viable protection mechanism.

An IsoMoD namespace may be constructed at run-time by an application core from an IsoMoD policy. This late binding of access control policy to code not only supports the separate maintenance of code and policy, but also supports the presentation of different views of the same application core to different extensions. 


\begin{tabular}{|l|l|}
\hline Access Right & Description \\
\hline Declared type target $C$ / Declared type subject $D$ \\
\hline extend & $D$ extends $C$ \\
\hline implement & $D$ implements $C$ \\
\hline Declared type target $C /$ Method subject $N$ \\
\hline catch & $N$ handles exception type $C$ \\
\hline cast & $N$ casts a reference to $C$ \\
\hline instanceof & $N$ checks if a reference is $C$ \\
\hline new & $N$ creates an instance of $C$ \\
\hline reflect & $N$ gets the Class object of $C$ \\
\hline new-array & $N$ creates an array of $C$ \\
\hline Similarly, cast-array, instanceof-array, reflect-array. \\
\hline Field target $F / M e$ thod subject $N$ \\
\hline get & $N$ reads $F$ \\
\hline put & $N$ writes $F$ \\
\hline Method target $M$ & Method subject $N$ \\
\hline invoke & $N$ invokes $M$ \\
\hline override & $N$ overrides $M$ \\
\hline
\end{tabular}

Figure 3.2: Access Rights

\begin{tabular}{|c|c|c|}
\hline \multicolumn{3}{|c|}{ Unary Predicates } \\
\hline final $(C)$ & abstract $(C)$ & interface $(C)$ \\
\hline public $(C)$ & package-private $(C)$ & \\
\hline \multicolumn{3}{|c|}{ Binary Predicates } \\
\hline subclass $(C, D)$ & superinterface $(C, D)$ & assignable $(C, D)$ \\
\hline extends $(C, D)$ & implements $(C, D)$ & \\
\hline
\end{tabular}

Figure 3.3: Built-in Predicates on Declared Types

\subsection{The IsoMod policy language}

The IsOMoD policy language provides a declarative and expressive means to specify the access control policy of an IsoMoD name space. An access is composed of three elements: (1) a subject, (2) an object, and (3) an access right. An object is also called a target to avoid confusion in the context of object-oriented programming. IsOMOD controls access to three kinds of targets: (a) declared types, (b) fields, and (c) methods. A declared type is either a class or an interface. For brevity, the word "class" is used as a synonym of "declared type". Every target is identified by a name visible in the IsoMoD namespace. A target can be accessed by exercising a fixed 


\begin{tabular}{|l|l|}
\hline formal-parameters $(M)$ & a list containing the formal parameters of method $M$ \\
\hline return-type $(M)$ & the return type of method $M$ \\
\hline field-type $(F)$ & the field type of field $F$ \\
\hline package $(C)$ & the package to which declared type $C$ belong \\
\hline equals $\left(O_{1}, O_{2}\right)$ & equality test \\
\hline
\end{tabular}

Figure 3.4: Built-in Functions on Declared Types, Methods, and Fields

set of access rights as outlined in Figure 3.2. A subject is either (a) a declared type whose name is defined in the IsoMoD namespace, or (b) a method declared in such a class.

An IsoMoD policy is made up of a finite number of policy clauses (or access control rules), each of which has the following general syntax:

$$
O \text { (allows|denies) }\left\{r_{1}, \ldots, r_{k}\right\}[\text { to } S][\text { (when|unless) } c \text { ] }
$$

In general, a policy clause tells whether or not a target $O$ grants (resp. denies) access rights $r_{1}, \ldots, r_{k}$ to a subject $S$. When the optional to-phrase is omitted, the rights are granted (resp. denied) categorically. An optional condition $c$ may also be supplied to specify when the policy clause is applicable (not applicable). The condition $c$ is a first-order predicate in $O$ and $S$. The IsoMoD policy language predefines a number of built-in connectives, predicates and functions for expressing complex applicability conditions. IsoMoD also provides a simple mechanism for policy programmers to define application-specific predicates and functions. A sample of built-in predicates is given in Figure 3.3. A sample of built-in functions is given in Figure 3.4.

Prior to the definition of a declared type [21, Section 5.3], its classfile is examined by the IsoMoD class loader for conformance to the corresponding IsoMoD policy. To this end, the set of all accesses in which the classfile (or one of its declared methods) is a subject is first collected. Each access is then checked according to the authorization algorithm outlined in Figure 3.5: the policy clauses are examined in the order they appear in the policy, and the authorization decision of the first applicable policy 
To decide if access $\langle S, O, r\rangle$ is granted by policy $P$ :

for each rule $R$ in policy $P$ do

if $R$ is relevant to $\langle S, O, r\rangle$ then

if $c$ is true then

if $R$ is a when-rule then

if $R$ is an allow-rule then return grant;

else $/ / R$ is a deny-rule

return deny;

else $/ / c$ is false

if $R$ is an unless-rule then

if $R$ is an allow-rule then return grant;

else $/ / R$ is a deny-rule

return deny;

return user-specified default;

Figure 3.5: Operational Semantics for Authorization

clause is then adopted. (A default authorization decision can be specified by the user of IsoMoD to handle the case when no policy clause applies.) In this process, if any access is denied by the policy, the definition of the declared type will not be authorized.

Simple as it is, the IsoMoD policy language is capable of expressing a rich family of access control policies, a topic to which we will now turn. 


\section{SAMPLE APPLICATIONS}

The goal of this chapter is to demonstrate the utility of the IsoMoD policy language through examples. The first example demonstrates how to selectively hide system services (Section 4.1). The second example illustrates how to systematically control the acquisition of references, and to do so in a way that accommodates evolving software configurations (Section 4.2). Finally, the ISOMOD policy language is employed to completely encode a capability policy language, thereby demonstrating the expressiveness of the policy language (Section 4.3).

\subsection{Selective hiding of system services}

IsoMOD can be used to enforce many of the BasicPermissions defined in the Java 2 platform [15]. For example, the getClassLoader permission controls whether untrusted code may acquire a ClassLoader reference from the platform library. The effects of denying this permission can be simulated by the IsOMOD policy below:

policy getClassLoader

default allow

method ClassLoader getParent

denies $\{$ invoke $\}$

method ClassLoader.getSystemClassLoader

denies $\{$ invoke $\}$

method Class.getClassLoader

denies $\{$ invoke $\}$

method Class Class.forName(String, boolean, ClassLoader)

denies $\{$ invoke $\}$

The policy begins with a header that identifies the policy name and asserts that the default authorization decision is to allow access (i.e., when no policy clause applies). 
Next come the policy clauses, which disallow invocation of all methods declared in the Java platform library that returns a ClassLoader. Notice that one may either specify a method target solely by its name (e.g., getClassLoader), or by both its name and its type signature (e.g., forName ${ }^{1}$ ).

The related createClassLoader permission controls whether untrusted code may create new instances of the ClassLoader class. In the Java 2 platform, security checks are embedded in the constructors of ClassLoader, SecureClassLoader and URLClassLoader for ensuring that the caller possesses the said permission. Denying the createClassLoader permission can be simulated with the following policy clause:

method C.M

denies $\{$ invoke $\}$

when constructor $(M)$ and subclass( $C$, ClassLoader $)$

Notice that this policy clause is more general than the ones aforementioned: it is applicable to any constructor $M$ of a class $C$ that is either ClassLoader or one of its subclasses (i.e., the predicate constructor tests if a method is a constructor, and the binary relation subclass is the reflexive transitive closure of the extends relation). Specifically, constructor invocation is denied. This rules out all means of creating ClassLoader instances.

The following is an alternative policy clause that achieves the same effect.

class $C$

denies $\{$ new $\}$

when $\operatorname{subclass}(C$, ClassLoader $)$

Rather than controlling the invocation of ClassLoader constructors, this policy clause directly disallows the creation of new ClassLoader instances.

\footnotetext{
${ }^{1}$ The forName method is denied because untrusted code may pass in a null classLoader reference to access the bootstrap ClassLoader.
} 
Most BasicPermissions defined in the Java 2 platform can be expressed declaratively by IsoMoD. There is, however, a clear software engineering advantage to the IsOMOD approach. Consider what is required in implementing and maintaining a Java 2 BasicPermission. One has to inspect the entire Java 2 platform library to identify all points of attack, and then interpose monitoring code at each point. When a vulnerability is found, library source code has to be modified. In the IsoMoD example above, an exhaustive audit of the platform library is still necessary, yet the maintenance path is far superior: the policy is expressed declaratively and maintained independently: e.g., in a policy file separate from the library source code.

The IsoMoD approach provides a way to enforce fine-grained access control policies not expressible by the Java 2 permission system. Suppose we are to prevent untrusted code from using the Reflection API to invoke methods, access fields and arrays, and create new object instances, but we want to permit the examination of class interfaces. The existing permissions defined in Java 2 are not suffcient for expressing this highly selective policy: the entire Reflection API must be turned on or off as a whole. However, there is no problem constructing IsOMOD policy clauses to selectively hide the following reflection services: (1) method invocation: Method.invoke; (2) field access: the Field.get/set family of methods; (3) array access: the Array.get/set family of methods; (4) object instantiation: Class .newInstance, Constructor.newInstance, Array .newInstance, Proxy .newProxyInstance; (5) subtyping: Proxy.getProxyClass.

\subsection{Systematic control of reference acquisition}

In the createClassLoader example discussed in Section 4.1, we could have formulated the following rule to deny the instantiation of new URLClassLoader instances:

method URLClassLoader. newInstance 
denies $\{$ invoke $\}$

We did not impose this policy clause because such a restriction is not part of the semantics of the createClassLoader permission. Yet, this observation reveals a general challenge in policy formulation. Suppose we want to eliminate all means by which untrusted code may acquire a ClassLoader instance (that is, either by retrieving an existing instance or by creating a new one). An exhaustive audit of the platform library must be conducted to ensure all means of leaking ClassLoader references are accounted for. Not only is this an error-prone approach, it does not account for many useful configuration management practices: What if non-standard platform extension libraries are installed? What if IsoMoD is used for isolating dynamically downloaded plug-ins of an extensible application? Platform extensions and application classes may expose additional means of leaking ClassLoader references. To ensure that the access control policy is bullet proof, even a minor perturbation of the software configuration will necessitate a re-audit of the software infrastructures. Such a practice is too costly to be feasible.

A major contribution of IsoMoD is that it offers an expressive and declarative policy language that addresses the aforementioned configuration management challenge in access control. We demonstrate this feature by producing an IsoMoD policy that systematically restricts the acquisition of ClassLoader references. To this end, we begin by exhaustively enumerating all means, excluding those involving array types, by which a reference of declared type $A$ may acquire a reference of type $C$ :

1. A declared type $A$ generates a reference of type $C$ when one of the following occurs: (a) $A$ creates an instance of $C$; (b) $A$ casts a reference to type $C$; (c) an exception handler in $A$ with catch type $C$ catches an exception.

2. A declared type $B$ shares a reference of type $C$ with declared type $A$ when one of the following occurs: (a) $A$ invokes a method declared in $B$ with return type 
$C$; (b) $A$ reads a field declared in $B$ with field type $C$; (c) $B$ writes a reference into a field declared in $A$ with field type $C$.

3. A declared type $B$ grants a reference of type $C$ to declared type $A$ when $B$ invokes a method declared in $A$, passing an argument via a formal parameter (including the pseudo-parameter this) of type $C$.

Based on the analysis above ${ }^{2}$, we formulate the following policy clauses to prevent untrusted code from acquiring a ClassLoader reference:

policy acquireClassLoader

default allow

class $C$

denies $\{$ new, cast, catch $\}$

when $\operatorname{subclass}(C$, ClassLoader $)$

field C.F

denies $\{$ get, put $\}$

when subclass(field-type $(F)$, ClassLoader)

method C.M

denies $\{$ invoke $\}$

when subclass(return-type $(M)$, ClassLoader)

method C.M

denies $\{$ invoke $\}$

when exists $A$ in parameter-types $(M): \operatorname{subclass(A,\text {ClassLoader)}}$

The first policy clause eliminates all means of generating ClassLoader references. The second and third policy clauses eliminate all means of sharing ClassLoader references. The last policy clause eliminates all means of granting ClassLoader references.

\footnotetext{
${ }^{2}$ For brevity, the analysis does not account for array types, but such an extension is straightforward.
} 
Built-in functions such as field-type, return-type and parameter-types are employed to specify fine-grained accessibility criteria. The use of existential quantification (exists) is also demonstrated.

The policy above systematically restricts the acquisition of ClassLoader instances. Neither policy reformulation nor source code auditing is necessary even if the configuration of the underlying system has evolved.

\subsection{Discretionary Capability Confinement}

This section demonstrates how IsoMoD can be used for enforcing a general-purpose capability type system, Discretionary Capability Confinement (DCC) [12]. A lightweight, statically enforceable type system, DCC supports the use of abstractly-typed object references as capabilities in a Java-like object-oriented programming language. A capability [8] is an object reference qualified by a set of access rights, where, the latter specify in what ways the underlying object can be accessed through the reference. Capabilities can be modeled in a language-based environment through a capability type system, in which every object reference is statically assigned a capability type that restricts access to the underlying object. In a sense, a capability type presents a restricted view of the object it types. In a Java-like object-oriented programming language, an object reference with a static interface type (or abstract class type) can be seen as a capability, because the typed reference only exposes a restricted view of the underlying object. This approach to modeling capabilities suffers from two problems: capability leakage and capability theft [34]. DCC is a minimal perturbation to Java for controlling capability propagation. In the following, the expressiveness of IsoMoD is demonstrated through a complete encoding of the DCC type system in the policy language. The focus here is IsoMoD and not DCC. Interested readers may consult [12] for more details of DCC. 
$(\mathcal{D C C} 1)$ Unless $B \triangleright A, A$ shall not invoke a static method declared in $B$.

$(\mathcal{D C C} 2)$ (i) $A$ can generate a reference of type $C$ only if $C \triangleright A$; (ii) $B$ may share a reference of type $C$ with $A$ only if $(C \triangleright A) \vee(A \bowtie B)$.

$(\mathcal{D C C} 3)$ If $A . m$ invokes $B . n$, and $C$ is the type of a formal parameter of $n$, then $(C \triangleright B) \vee(A \bowtie$ $B) \vee(B \triangleright m \wedge C \triangleright m)$.

$(\mathcal{D C C} 4)$ A method $m$ may invoke another method $n$ only if $n \triangleright m$.

$(\mathcal{D C C} 5)$ If $A$ is a subtype of $B$, then $B \triangleright A$.

$(\mathcal{D C C} 6)$ Suppose $B . n$ is overridden by $B^{\prime} . n^{\prime}$. Then (i) $n^{\prime} \triangleright n$; (ii) if the method return type is $C$, then $(C \triangleright B) \vee\left(B \triangleright B^{\prime}\right)$; (iii) if $C$ is the type of a formal parameter, then $\left(C \triangleright B^{\prime}\right) \vee\left(B \bowtie B^{\prime}\right)$.

$(\mathcal{D C C} 7)$ If $A$ is a subtype of $B$, then $B: \triangleright A$.

$(\mathcal{H M S 1}) \mathrm{T}: \mathcal{D}$.

$(\mathcal{H M S} 2)$ If $\mathcal{D}: \triangleright \mathcal{E}$, then $\mathcal{D} \triangleright \mathcal{E}$.

$(\mathcal{H M S 3})$ If $(\mathcal{D}: \mathcal{E}) \wedge\left(\mathcal{D}^{\prime} \triangleright \mathcal{E}\right)$, then $\left(\mathcal{D} \triangleright \mathcal{D}^{\prime}\right) \vee\left(\mathcal{D}^{\prime} \triangleright \mathcal{D}\right)$.

Figure 4.1: DCC Type Constraints

In DCC, the space of declared types (i.e., class and interface) is partitioned into a finite number of confinement domains, so that every declared type belongs to exactly one confinement domain. We write $l(C)=\mathcal{D}$ when declared type $C$ is assigned to confinement domain $\mathcal{D}$. The confinement domains are partially ordered by a dominance relation $\longrightarrow$. We say that domain $\mathcal{D}$ dominates domain $\mathcal{E}$ when $\mathcal{E} \rightarrow \mathcal{D}$. Together, domain membership and dominance induce a natural pre-ordering of declared types: if $l(B)=\mathcal{E}, l(A)=\mathcal{D}$, and $\mathcal{E} \triangleright \mathcal{D}$, then we write $B \triangleright A$, and say that $B$ trusts $A$. The intuition behind these definitions is that, if $C$ trusts $A$, then $A$ may freely acquire a reference of static type $C$. Otherwise, $C$ is said to be a capability for $A$. Capability acquisition is carefully restricted in DCC. We also write $A \bowtie B$ when $A \triangleright B$ and $B \triangleright A$ hold simultaneously. We postulate that there is a root domain $T$ which is dominated by every domain. 
To control capability granting, associated with every method $m$ is a domain label $l(m)$, called the capability granting policy of $m$. Intuitively, the capability granting policy $l(m)$ dictates what capabilities may be granted by $m$, and to which declared types $m$ may grant a capability. (We write $m \triangleright n, m \triangleright A$ and $A \triangleright m$ for the obvious meaning.)

A second partial ordering $: \boldsymbol{\nabla}$ on domains is postulated. We say that $\mathcal{D}$ strongly dominates $\mathcal{E}$ when $\mathcal{E}: \mathcal{D}$. As we shall see, strong dominance controls whether subtyping is allowed across domain boundaries. This helps to establish mutually exclusive roles. As before, we write $B: \triangleright A$ when $l(B)=\mathcal{E}, l(A)=\mathcal{D}$, and $\mathcal{E}: \triangleright \mathcal{D}$.

Figure 4.1 enumerates the type constraints of DCC as specified in [12]. The complete set of DCC type constraints has been successfully encoded as an IsoMOD policy, which is displayed in Appendix A. Behind the policy of Appendix A is a number of assumptions. As in [12], we assume that domain membership and capability granting policies are embedded in Java classfiles via the JDK 1.5 metadata facility. Domains are represented by specially annotated interfaces, and the dominance and strongly dominance relations are encoded, respectively, by the subinterfacing relation and JDK 1.5 annotations. Domain-specific functions and predicates have been defined to examine these annotations. In the following we will examine aspects of this encoding that illustrate further features of IsoMoD.

Consider the following type constraint from Figure 4.1:

$(\mathcal{D C C} 2)$ (i) $A$ can generate a reference of type $C$ only if $C \triangleright A$; (ii) $B$ may share a reference of type $C$ with $A$ only if $C \triangleright A \vee A \bowtie B$.

In this constraint, the first clause denies the generation of capabilities, and the second clause denies the sharing of capabilities with reference types belonging to a different confinement domain. This constraint can be encoded as the following IsoMoD policy clauses:

class $C$ 
denies $\{$ catch, cast, new $\}$ to method $A . M$

unless trusts $(C, A)$

method $B . N$

denies $\{$ invoke $\}$ to method $A . M$

unless trusts(return-type $(N), A)$ or $($ trusts $(A, B)$ and trusts $(B, A))$

field $B . F$

denies $\{$ get $\}$ to method A.M

unless trusts $($ field-type $(F), A)$ or $(\operatorname{trusts}(A, B)$ and trusts $(B, A))$

field B.F

denies $\{$ put $\}$ to method $A . M$

unless $\operatorname{trusts}(A$, field-type $(F))$ or $(\operatorname{trusts}(A, B)$ and $\operatorname{trusts}(B, A))$

Two additional features of ISOMOD are demonstrated in the above policy clauses. Firstly, IsoMoD provides a syntax (i.e., to) for qualifying to which subject a policy clause applies. As capability acquisition is permitted for some subjects but not others, this discrimination enables fine-grained access control. Secondly, IsoMoD supports user-defined predicates and functions for modeling domain-specific relations. For example, trusts is a user-defined predicate for representing the binary trust relation between declared types.

Let us consider another type constraint from Figure 4.1:

$(\mathcal{H M S} 3)$ If $(\mathcal{D}: \triangleright \mathcal{E}) \wedge\left(\mathcal{D}^{\prime} \triangleright \mathcal{E}\right)$, then $\left(\mathcal{D} \triangleright \mathcal{D}^{\prime}\right) \vee\left(\mathcal{D}^{\prime} \triangleright \mathcal{D}\right)$

This constraint is the soul of a property known as hereditary mutual suspicion [12], which enforces a strong form of separation of duty $[6,19]$, so that collusion between mutually exclusive roles is severely restricted. The constraint mandates that, given an arbitrary domain $\mathcal{E}$, some form of dominance relation must exist between a domain $\mathcal{D}$ strongly dominated by $\mathcal{E}$ and a domain $\mathcal{D}^{\prime}$ dominated by $\mathcal{E}$. An IsoMon encoding of it is given below: 


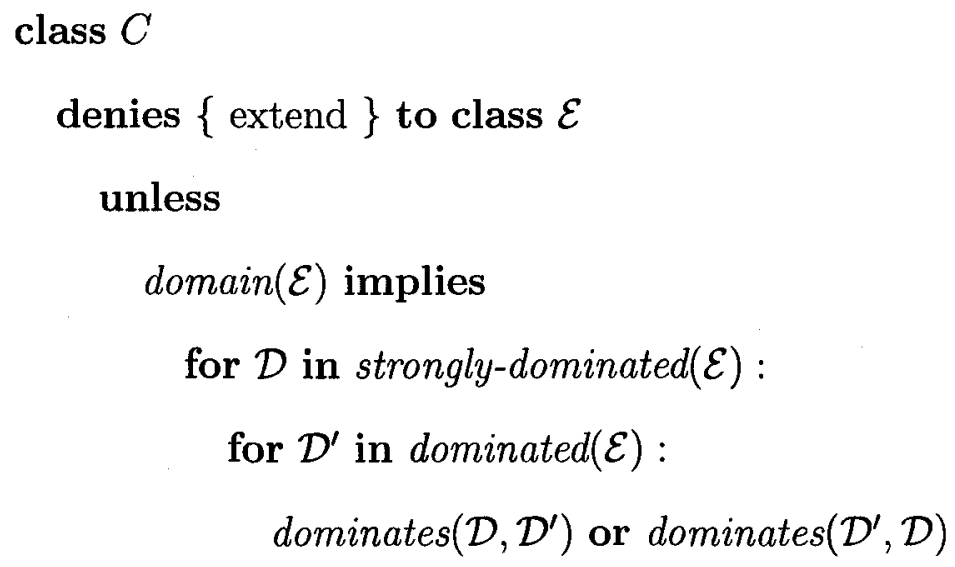

Our goal is to check $(\mathcal{H} \mathcal{M S} 3)$ exactly once for every domain $\mathcal{E}$. To this end, we observe that, at the bytecode level, every declared type extends exactly one superclass, with java.lang.Object being the only, uninteresting exception. We therefore "schedule" the verification of $(\mathcal{H M S} 3)$ to occur when $\mathcal{E}$ extends some dummy class $C$. The same technique is used in the encoding of $(\mathcal{H} \mathcal{M S} 1)$ and $(\mathcal{H M S} 2)$ (see Appendix A).

Besides DCC, we have also completely encoded the class-based access control mechanism of Java [21, Section 5.4.4] (i.e., public, protected, private, etc) as an IsoMoD policy (Chapter 6). These examples demonstrate the expressiveness and versatility of the IsoMoD policy language. 


\section{IMPLEMENTATION EXPERIENCE}

IsoMoD has been fully implemented in approximately 10,000 lines of pure Java code. This chapter reports the implementation experience. The technical challenges encountered and the solutions adopted to address these challenges are discussed. Enough details of the IsoMoD load-time verification mechanism are given so that its design can be reused in future work involving load-time type checking.

This chapter is organized as follows. Section 5.1 provides a high-level outline of the implementation strategy, and highlights the technical challenges it is designed to address. Section 5.2 describes the data structure incrementally constructed by IsoMoD to facilitate policy enforcement. Section 5.3 provides an in-depth treatment of the load-time verification algorithm, and discusses how the algorithm accomplishes the design goals. Section 5.4 describes the process of policy evaluation and access authorization. The chapter concludes with a summary in Section 5.5.

\subsection{Design rationales}

This section explores the technical challenges our implementation strategy attempts to address. We begin by identifying the challenges:

1. Efficiency. Class loading and policy evaluation incur a significant link-time overhead, slow down application start-up, and should thus be minimized.

2. Early enforcement. Class definition [21, Section 5.3.5] is irrevocable. Policy enforcement must be complete before a classfile is converted into a Class object.

3. Circularity. Circular dependency between type interfaces may arise from forward references. Policy evaluation must handle circularity gracefully.

4. Attribution correctness: Policy violation should be attributed to the offending classfiles (i.e., subjects) rather than the offended classfiles (i.e., targets). 
Only the definition of the subjects should be denied.

Initially we considered three possible designs, and eventually adopted one that adequately addresses all of the aforementioned technical challenges. The difference between the three designs lies in when external dependencies are resolved. Specifically, before a class is defined, its corresponding classfile is preloaded and screened for policy violation. Policy checking may involve the examination of external classes, as the following example (taken from Section 4.2) illustrates:

method C.M

denies $\{$ invoke $\}$ when subclass(return-type $(M)$, ClassLoader)

Checking whether a declared type $A$ conforms to this policy clause involves examining both $C$ and return-type $(M)$. These latter declared types may not have been preloaded yet. The three designs differ in how this situation is handled.

Design \#1: Eager preloading. Preload $C$ and return-type $(M)$ right away. While this design is easy to implement, the resulting class loading overhead will likely be prohibitive.

Design \#2: Constraint maintenance. Lazy preloading can be achieved by constraint maintenance. That is, the definition of $A$ is authorized right away, but the policy clause is recorded as a proof obligation, the discharging of which is deferred until $C$ and return-type $(M)$ are preloaded at a later time. This design eschews eager preloading, but its implementation is complex. This design has been adopted by Sun's implementation of the JVM for enforcing Temporal Namespace Consistency [20]. The most serious problem with this approach is its inability to correctly attribute policy violation to the offender. When the checking of the proof obligation fails at the time 
$C$ or return-type $(M)$ is preloaded, the only thing a system could do is to deny the definition of $C$ and return-type $(M)$, an incorrect attribution of policy violation.

Design \#3: Three-stage, lightweight preloading. Preloading is divided into three stages (Figure 5.1), which incrementally constructs and maintains a lightweight representation of the classfile being preloaded. In the first stage, references to the classfile's supertypes are resolved, and all "extend" and "implement" accesses are checked. In the second stage, type references appearing in the type interface of a classfile are resolved, and then all "override" accesses are checked. In the third stage, type references appearing in the remaining external accesses are resolved, and then those accesses are checked. This design performs shallow preloading eagerly, but maintains lightweight type mirrors to anticipate deep preloading. This design implements a lazy preloading strategy, carefully breaks circularity, and correctly attributes policy violations to the offending classfiles. The preloading algorithm is detailed in Figure 5.1. This design is informed by previous work in modular bytecode verification in the presence of lazy, dynamic linking $[13,11]$.

The rest of this chapter provides details concerning Design \#3, which was adopted in the implementation of IsoMoD.

\section{$5.2 \quad$ Type mirrors}

This section describes the lightweight representation of declared types created by IsOMOD. This lightweight representation is called a mirror in the programming language literature [5]. More specifically, IsoMoD maintains a mirror object for each declared type, method, field, and external access. The following presents the layout of these data structures and the incremental process by which they are constructed. 
Stage-I Preloading of Declared Type $C$

1. Retrieve the classfile of $C$.

2. Perform stage-I preloading on the supertypes of $C$. Circular subtyping is detected.

3. Check the "extend" and "implement" accesses of $C$.

4. Cache a lightweight representation of $C$, recording its type interface and the remaining external accesses.

Stage-II Preloading of Declared Type $C$

1. Perform stage-I preloading on $C$.

2. Perform stage-II preloading on the supertypes of $C$.

3. Perform stage-I preloading on the return types and parameter types of methods declared in $C$, and the field types of fields declared in $C$.

4. Check the "override" accesses of $C$.

Stage-III Preloading of Declared Type $C$

1. Perform stage-II preloading on $C$.

2. Perform stage-III preloading on the supertypes of $C$.

3. Perform stage-II preloading on those type references appearing in the targets of the remaining external accesses associated with $C$.

4. Check the remaining external accesses of $C$.

5. Authorize the definition of declared type $C$.

Figure 5.1: Preloading Algorithm

\subsubsection{Structure of mirrors}

A classfile is a file format used by Java to represent object code in a file system. Each classfile describes a declared type (i.e., a class or an interface), including its methods, fields, and bytecode instructions. In order to perform verification, the preloading process converts a classfile into its respective mirror object, called a declared type mirror, or simply type mirror, the structure of which is described below. Notice that the following describes the data structures as they are fully constructed. The intermediate steps are left to Section 5.2.2.

Type Mirror A type mirror records the type interface of a declared type.

Class name The name of this declared type

Super class* The type mirror representing the direct super class of this declared type

Interfaces* The type mirrors of the interfaces directly implemented by this 
declared type

Declared methods* The method mirrors of the methods (including constructors) declared in this declared type

Declared fields ${ }^{*}$ The field mirrors of the fields declared in this declared type

External accesses* The access mirrors corresponding to the external accesses made by this declared type (e.g., as reflected in the bytecode instructions)

Method Mirror A method mirror describes the signature of a method.

Method name Name of this method

Return type $^{* *}$ The type mirror representing the return type of this method

Parameter types** The type mirrors representing the parameter types of this method

Defining class* The type mirror of the declared type in which this method is declared

Field Mirror A field mirror describes the signature of a field.

Field name Name of this field

Field type ${ }^{* *}$ The type mirror representing the type of this field

Defining class* The type mirror of the declared type in which this field is declared

Access Mirror An access mirror represents an external access, which is made up of a triple $\langle$ subject, object, right $\rangle$. Such a data structure is recorded to facilitate lazy verification.

Subject* The subject of this external access, which can be either a type or method mirror 
Object $^{* * *}$ The object (i.e., target) of this external access, which can be either a type, method or field mirror

Right A particular right exercised by this access (Figure 3.2)

\subsubsection{Staged construction of mirrors}

This subsection describes the incremental process by which the aforementioned mirrors are constructed. The mirror objects contain references to other mirror objects. These references are indicated by one or more asterisks $\left({ }^{*},{ }^{* *}\right.$, or $\left.{ }^{* * *}\right)$ in Section 5.2.1. For example, a type mirror contains a reference to the mirror of its direct super class; an access mirror contains a reference to the mirror object representing the target of the external access. During the preloading process, some of the references are not fully resolved, because the targets of the references have not been constructed (or preloaded) yet. In these cases, a symbolic reference (e.g., a string) is recorded in place of the actual mirrors. The following explains when mirrors are constructed and how symbolic references are resolved.

Stage-I This stage initiates the construction of mirror objects for a given classfile. Another goal is to incrementally construct a type mirror hierarchy to facilitate $s u b$ typing queries. When a declared type is preloaded in Stage-I, mirrors of methods declared in that declared type are created. At this point, the bytecode of the method body is scanned, and all external accesses are recorded as access mirrors. If the same access occurs multiple times in the method body, only a single instance is recorded. The newly created method mirror is linked to the type mirror, and vice versa. Similarly, a field declared in the declared type has its respective field mirror constructed and properly linked. The direct supertypes (super class and super interfaces) are resolved into their respective type mirrors through recursive Stage-I Preloading (Figure 5.1, Stage I, Step 2). In short, all the mirror references annotated with a single 
asterisk $\left({ }^{*}\right)$ in Section 5.2.1 are resolved in this stage. By the end of Stage-I, the basic structure of the type mirror is established, its external accesses recorded, and the part of the type mirror hierarchy above this type mirror fully constructed.

Stage-II This stage is responsible for resolving the symbolic references appearing in method and field mirrors: i.e., the ones annotated with double asterisks $\left(^{* *}\right)$ in Section 5.2.1. The goal of this stage is to make the type interface of a given type mirror fully usable. Specifically, the method and field mirrors of the type mirror are examined. Symbolic references corresponding to the return type and parameter types of a method mirror are resolved through Stage-I Preloading. The method mirror is updated, by linking the respective components to their mirror counterparts. The field types of field mirrors are updated in a similar fashion. Notice that Stage-II Preloading is performed on the mirrors of supertypes before it is performed on a given type mirror (Figure 5.1, Stage II, Step 2). This means, not only are the mirrors of methods and fields declared in the type mirror fully constructed in Stage II, the mirrors of those methods and fields inherited by the type mirror are also fully constructed by then. By the end of Stage-II, the type interface of the type mirror is fully constructed to facilitate type interface queries.

Stage-III This stage is responsible for resolving the targets of external accesses: i.e., those marked by triple asterisks $\left(^{* * *}\right)$ in Section 5.2.1. The goal is to resolve the symbolic references embedded in access mirrors, so that the external accesses can be authorized prior to class definition [21, Section 5.3.5]. To this end, the collection of access mirrors associated with a given type mirror are scanned. For every access mirror, each of its targets is examined. If the target is a type reference (e.g., as in the case of a "new" access), the reference is resolved by Stage-II Preloading. If the target is a method (resp. field) reference (e.g., as in the case of an "invoke" access), then such a reference will be of the form $c . m$ (resp. c.f), where $c$ is a symbolic reference to a 
declared type, and $m$ (resp. $f$ ) is a method signature (resp. field signature) consisting of a method name plus return type and parameter types (resp. field name plus field type). The type reference $c$ is resolved into a type mirror $C$ by Stage-II Preloading, after which the proper method mirror (resp. field mirror) is located in either $C$ or one of $C$ 's supertype mirrors. This resolution process closely parallels that of $[21$, Section 5.4.3]. This final step concludes the staged construction process for a type mirror.

An example of this staged construction process is given in Figure 5.2.

\subsection{Staged verification}

Not all external accesses are verified at once (Figure 5.1). The "extend" and "implement" accesses are discharged in Stage I, "override" in Stage II, and the rest in Stage III. Why is the verification process divided into three stages? And why is a given check scheduled to occur in a specific stage? These are the questions the present section attempts to answer. As we shall see, the three-stage design facilitates lazy preloading and gracefully handles circularity.

Stage-I Recall that this stage gathers subtyping information for a given declared type. Such subtyping information must be verified before it can be queried. Firstly, circular subtyping is detected by checking if a type mirror argument re-appears in the call chain of recursive Stage-I Preloading. Secondly, access mirrors corresponding to "extend" and "implement" rights are verified in this stage against the current policy. The completion of this stage ensures that subtyping queries are supported by properly verified subtyping information.

Notice as well that circular Stage-I Preloading is prevented because circular subtyping is ruled out. 


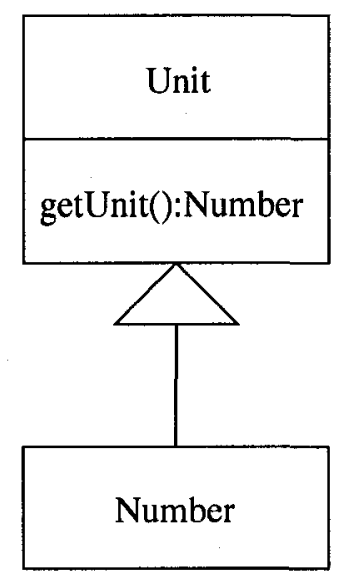

Classfile Unit is requested to be loaded

1. Stage-III Preloading is performed on Unit

1.1. Stage-II Preloading is performed on Unit

1.1.1. Stage-I Preloading is performed on Unit

1.1.1.1. Mirror of Unit is constructed

1.1.1.2. Super class of Unit is determined to be Object

1.1.1.3. Stage-I Preloading is performed on Object

1.1.1.4. Mirror of Unit is linked to the mirror of super class Object

1.1.2. Stage-II Preloading is performed on super class Object

1.1.3. Return type of method Unit.getUnit() is determined to be Number

1.1.4. Stage-I Preloading is performed on Number

1.1.4.1. Mirror of Number is constructed

1.1.4.2. Super class of Number is determined to be Unit

1.1.4.3. Stage-I Preloading is performed on Unit

1.1.4.3.1. Stage-I Preloading is already performed on Unit

1.1.4.4. Mirror of Number is linked to the mirror of super class Unit

1.1.5. Mirror of Unit.getUnit() is linked to mirror of return type Number

2. Definition of Unit is authorized

Figure 5.2: Preloading Example 
Stage-II Recall that by the end of Stage II, the type interface of a type mirror is fully constructed to support type interface queries. The type interface of a declared type extends the type interfaces of its supertypes through inheritance and method overriding. To ensure type extension is proper, the "override" accesses are checked in this stage for policy compliance. This ensures that type interface queries are supported by a properly verified type interface.

Two kinds of recursive preloading are performed in this stage. Firstly, Stage-II Preloading is performed on the type mirrors of the supertypes. This does not lead to circular preloading because circular subtyping has already been ruled out in Stage I. Secondly, Stage-I Preloading is invoked on type references embedded in method and field mirrors. This does not lead to circular preloading because Stage-I Preloading never invokes Stage II Preloading.

Stage-III Recall that the third and final stage is responsible for resolving the symbolic references embedded in the targets of access mirrors, so that the latter can be discharged. Resolution is achieved by Stage-II Preloading. As a result, the following queries are supported:

- Type interface queries can be applied to the targets of access mirrors.

- Subtyping queries can be applied to the type mirrors embedded in the type interface of the above access targets.

Observe that these are the very queries that the IsoMoD policy language is designed to support. The remaining access mirrors are therefore ready for verification.

Again, two kinds of recursive preloading is performed in this stage. Firstly, StageIII Preloading is performed on the type mirrors of the supertypes. This does not lead to circular preloading because of the absence of circular subtyping. Secondly, Stage-II Preloading is invoked on type references embedded in the targets of access mirrors. 
This, again, does not lead to circular preloading because Stage-II Preloading never initiates Stage-III Preloading.

Efficiency Observe from the above discussion that access mirrors are only checked when it is absolutely necessary to do so. To check an access mirror, type interface queries will be made against the target, and thus it undergoes Stage-II Preloading. Similarly, subtyping queries will be made against the type references embedded in the type interface of the access target, so Stage-II Preloading guarantees that such references are resolved via Stage-I Preloading. In this way, preloading is minimized to improve preloading efficiency.

Circularity The staged preloading and verification process gracefully handles the two possible types of circularity. Subtyping circularity is managed in Stage I by ensuring that no circular subtyping occurs. Circularity arising from forward referencing is handled by stratification of the preloading process: Stage I never invokes Stage II and III; Stage II never invokes Stage III. This effectively breaks any possibility of circularity. This fact is demonstrated in the example preloading trace in Figure 5.2.

\subsection{Policy evaluation}

When an access mirror is checked against an IsoMoD policy, the policy clauses are examined in turn. IsoMoD evaluates each of the policy clauses to determine if the clause is applicable to the access. Applicability is specified in the IsoMoD policy language via the when/unless construct, which states a binary relation between the subject and the target. This binary relation is expressed in the form of a firstorder formula with free variables denoting the subject and the target. Determining the applicability of a policy clause involves binding the subject and target variables, respectively, to the subject and target mirrors, and subsequently deciding if the mirror 
hierarchy that has been preloaded so far is a model for the formula. That is, the firstorder formula is evaluated against the subtyping and type interface information that IsoMoD has collected so far. The following outlines some of the standard language processing techniques that have been applied to implement policy evaluation.

Policy clauses, and especially their applicability conditions, are represented internally as abstract syntax trees (ASTs). A standard object-oriented encoding of ASTs has been adopted [40]:

- For every syntactic category (i.e., non-terminal) $\langle A\rangle$, an abstract class $A$ is declared to model that syntactic category.

- For every possible production $(P)$ of the form

$$
\langle A\rangle::=x_{1} \ldots x_{k}
$$

in the abstract syntax, a concrete subclass $P$ of $A$ is declared to model that production.

- For every non-terminal $\langle B\rangle$ appearing on the right-hand side of a production $(P)$, a field of type $B$ is declared in the concrete class $P$.

This scheme can be illustrated by the following example. Consider the following fragment of the abstract syntax of the IsoMoD policy language:

$$
\begin{array}{rlr}
\langle\text { formula }\rangle & ::=\langle\text { formula }\rangle \text { and }\langle\text { formula }\rangle & (\text { And }) \\
& :=\operatorname{not}\langle\text { formula }\rangle & (\text { Not }) \\
& ::=\ldots
\end{array}
$$

The following Java classes are declared.

public abstract class Formula $\{\ldots\}$

public class AndFormula extends Formula \{ 


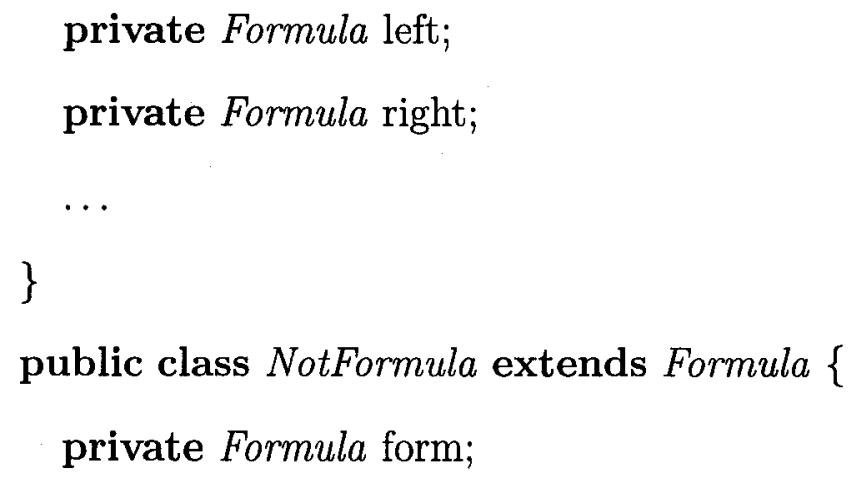

Evaluation of an applicability formula is conducted by an environment-passing interpreter [14], which is conceptually a procedure expecting three parameters:

- an AST, representing the first-order formula to be evaluated

- a binding environment, mapping variable names to mirror objects

- a handle to the type mirror hierarchy, through which subtyping and type interface queries can be evaluated

The evaluation procedure processes the AST recursively, in a syntax-directed manner: to compute the truth value of a formula, the sub-formulas are first evaluated recursively. The binding environment is extended when a quantifier scope begins (i.e., in the body of a for or exists). Built-in predicates and functions are interpreted as appropriate queries to the type mirror hierarchy.

\subsection{Summary}

This chapter presents an implementation strategy for IsoMoD that addresses such technical challenges such as performance and circularity. The staged design promotes lazy preloading of mirror objects by performing preloading and verification 
only when they are absolutely necessary. As well, Stage-I of the preloading process detects subtyping circularity, while Stage-II and Stage-III manage circularity arising from forward referencing. Policy evaluation is performed by a syntax-directed, environment-passing interpreter.

A natural question to ask is whether this implementation strategy delivers its performance promise, a topic to which we will now turn. 


\section{PERFORMANCE EVALUATION}

In this chapter, the performance of ISOMOD is evaluated by experiments and analysis. Section 6.1 defines the goals of this study, namely tractability, overhead composition and overhead distribution. Section 6.2 describes the methodology that was adopted in performing the experiments. Section 6.3 describes the first experiment that was conducted to discover the overhead incurred by IsoMoD. Section 6.4 discusses the analysis that was conducted on the first experiment to discover the components making up the overhead of IsOMOD. Section 6.5 describes the experiment that was conducted on a longer executing system, demonstrating that the overhead would amortize away. Section 6.6 summarizes the results from the experiments and analysis.

\subsection{Introduction}

This chapter reports the results of experiments conducted for profiling the performance characteristics of ISOMOD. The goal of this study is threefold:

1. Tractability: To quantify the performance overhead incurred by executing an application through IsoMOD.

2. Overhead Composition: To identify the major components of the IsoMoD implementation which contribute to the overhead incurred.

3. Overhead Distribution: To characterize the way in which the overhead incurred by IsOMOD is distributed over the life cycle of an application.

These characteristics are important in validating IsoMOD as a viable approach to software security.

The Tractability Study in Section 6.3 measures the overhead incurred by using IsoMoD. A quantifiable result was obtained through this study by comparing the running times of a test suite with and without IsoMoD 
The analysis described in Section 6.4 further examined the results gathered from the Tractability experiment. The purpose of this analysis was concerned with discovering the makeup of IsoMoD's overhead.

The above analysis suggested that the majority of the overhead occurred during application startup. Section 6.5 describes an experiment designed to discover how a longer executing system would experience overhead as opposed to that of a shorter lived execution.

The results of the experiments and analysis aided in validating IsoMoD as a viable approach to software security. The first experiment demonstrated that the overhead incurred by IsOMOD was reasonable and manageable. The analysis of this experiment discovered IsoMoD's overhead to be comprised of two major parts. The first part consisted of maintaining the type mirror hierarchy and the second was authorization of the access mirrors. The second experiment demonstrated that a longer executing system caused IsoMoD's overhead to amortize away. That is, a longer executing system experienced less of an overhead encumbrance as opposed to that of a shorter lived system.

\subsection{Methodology}

The experiments were conducted on a system with the following characteristics:

- $3.0 \mathrm{GHz}$ Pentium 4

- 1 Gigabyte of RAM

- Fedora Core 4 Linux Operating System

- Java Standard Edition, version 1.5

Accompanying software included BCEL (Byte Code Engineering Library), version 5.1 . 
In our testing, we used a test suite consisting of six open source Java applications (Figure 6.1). These applications were chosen because they could be executed in batch mode without real-time interaction from users. The applications were tested under five configurations. The first configuration, Control, runs an application in a bare JVM. The other four configurations consisted of loading an application with an IsoMoD class loader with one of four ISOMOD policies. The policies used in the configurations consisted of:

$N U L L$ Contains no policy clauses, and the default authorization decision is to allow all accesses.

AllowAll Contains policy clauses allowing access for every right occurring within the JVM. See Figure 6.2.

$D C C$ An IsoMoD encoding of the Discretionary Capability Confinement capability type system. See Appendix A.

$J A C$ Encoding of the Java access control mechanism as specified in [21, Section 5.4.4]. See Figure 6.3.

Five trials ${ }^{1}$ were repeated for each configuration to account for variability in the Operating System. Factors such as process scheduling and context switching may have contributed variations to the results. The average running time (in seconds) was recorded for each configuration.

In Section 6.5, the Java application JavaTar was used for testing under ten configurations. The configurations consisted of loading the application with the IsoMoD class loader with the $D C C$ policy and input data varying in size from $24 \mathrm{~KB}$ to $495 \mathrm{MB}$. The $D C C$ policy was chosen for its complexity, which would incur significant overhead.

\footnotetext{
${ }^{1}$ five trials were used due to the small standard deviation and variance in the experimental results as shown in Appendix B.
} 


\begin{tabular}{|c|l|c|}
\hline Name & Description & Number of Classes \\
\hline ZipDiff & Utility to compare the contents of two zip files & 8 \\
\hline JavaTar & A TAR compression program written in Java & 43 \\
\hline JavaCC & Sun's Compiler-Compiler & 135 \\
\hline SableCC & A compiler-compiler & 263 \\
\hline JRuby & A Java implementation of the Ruby language & 473 \\
\hline Kawa & A Scheme interpreter written in Java & 746 \\
\hline
\end{tabular}

Figure 6.1: Benchmarking Suite

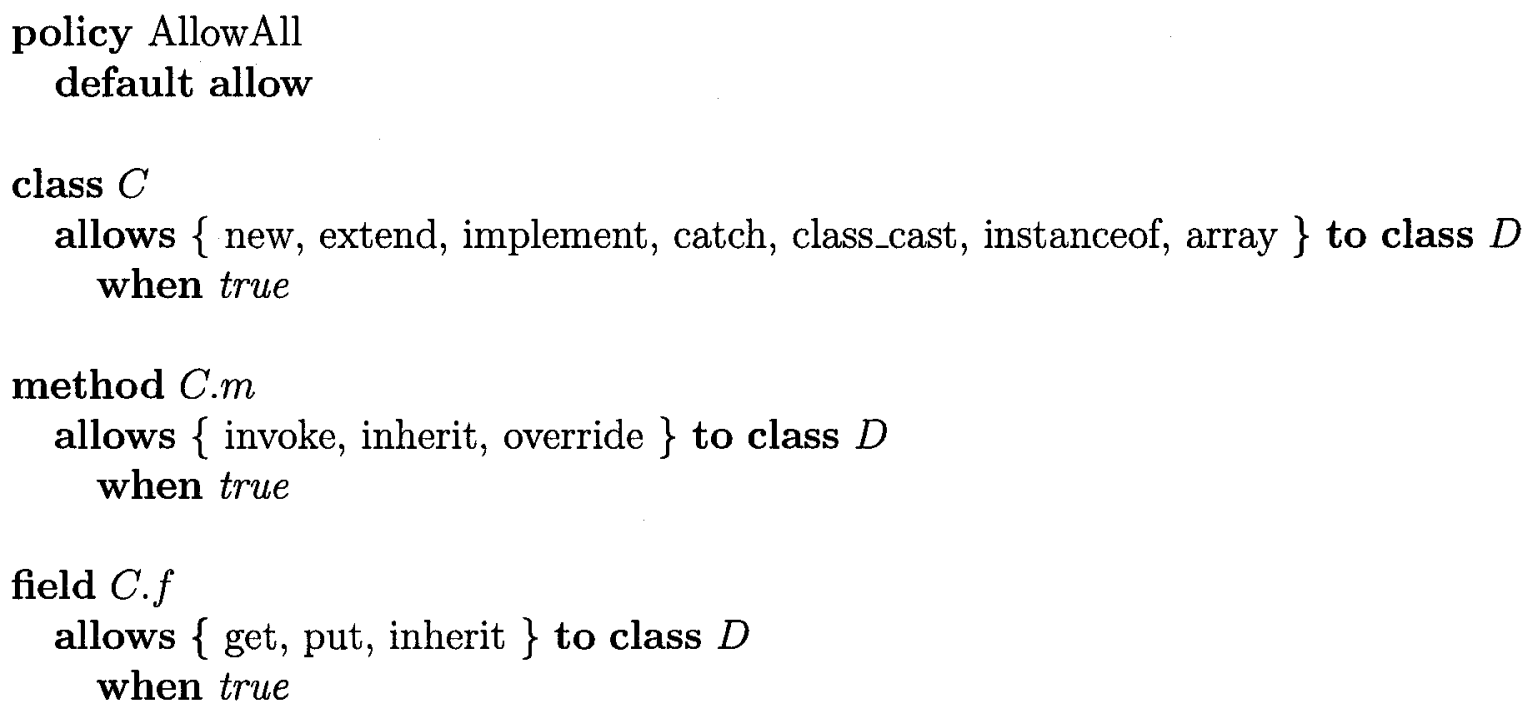

Figure 6.2: AllowAll Policy 


\section{policy JAC \\ default allow}

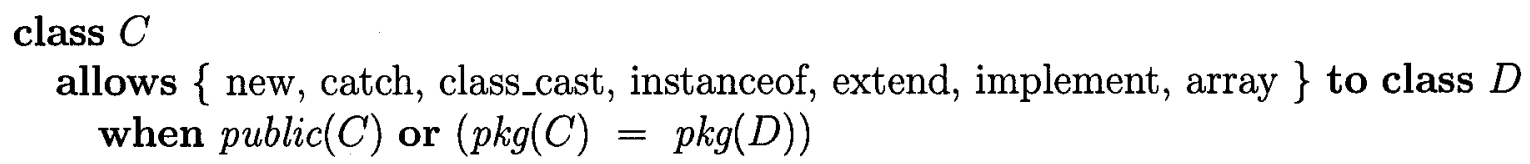

\section{field $C . F$}

allows $\{$ get, put $\}$ to class $D$

when

$$
\begin{aligned}
& (p u b l i c(C) \text { or }(p k g(C)=p k g(D))) \\
& \text { and }((p u b l i c(F) \text { or }(\text { protected }(F) \text { and } \operatorname{subclass}(D, C))) \\
& \quad \text { or }(((\operatorname{protected}(F) \text { or pkg-private }(F)) \text { and }(p k g(C)=p k g(D))) \text { or } \\
& \quad(\operatorname{private}(F) \text { and }(C=D))))
\end{aligned}
$$

\section{method C.M}

allows $\{$ invoke $\}$ to class $D$

\section{when}

$$
\begin{aligned}
& (\operatorname{public}(C) \text { or }(p k g(C)=p k g(D))) \\
& \text { and }((p u b l i c(M) \text { or }(\text { protected }(M) \text { and } \operatorname{subclass}(D, C))) \\
& \quad \text { or }(((\operatorname{protected}(M) \text { or pkg-private }(M)) \text { and }(p k g(C)=p k g(D))) \text { or } \\
& \quad(\operatorname{private}(M) \text { and }(C=D))))
\end{aligned}
$$

Figure 6.3: Java Access Control (JAC) Policy 


\subsection{Tractability}

This experiment quantified the overhead incurred by the IsoMoD class loader. A classfile loaded by the ISOMOD class loader is subjected to the verification process described previously. During this verification process, the complete collection of access mirrors is collected. These access mirrors are authorized against a policy. A classfile that passes all stages of verification is turned into a Class object and loaded into the JVM.

The results of the experiment are shown in Figure 6.4. This figure describes the run-time overhead incurred by the ISOMOD class loader for each application under the five configurations. From this figure it can be seen that the $N U L L$ configuration incurred the least amount of overhead, while $D C C$ incurred the most. The other two configurations, AllowAll and $J A C$ incurred almost equal amounts of overhead. The values of running time are shown in seconds and represent the total time for an execution.

A number of observations can be made about these results. First, the graph describes how much overhead is incurred when an application executes through the IsoMoD class loader. Regardless of whether a policy is enforced or not, IsoMoD incurs some overhead. This fact is demonstrated by the NULL configuration, where no policy clauses exist in the policy. Second, as the policies become more complex, the run-time overhead increases, as shown by the $D C C$ configuration. With the introduction of more policy clauses, and more complex applicability conditions, more time must be given to authorize the accesses described within the mirror. Third, the total overhead for any application never exceeded three seconds. In the process of a longer executing application such as SableCC which executed for just over ten seconds, three seconds of additional overhead may be considered reasonable.

Overhead while executing through IsoMoD cannot be avoided, but it has been demonstrated in the experimental results that the overhead is manageable and reason- 


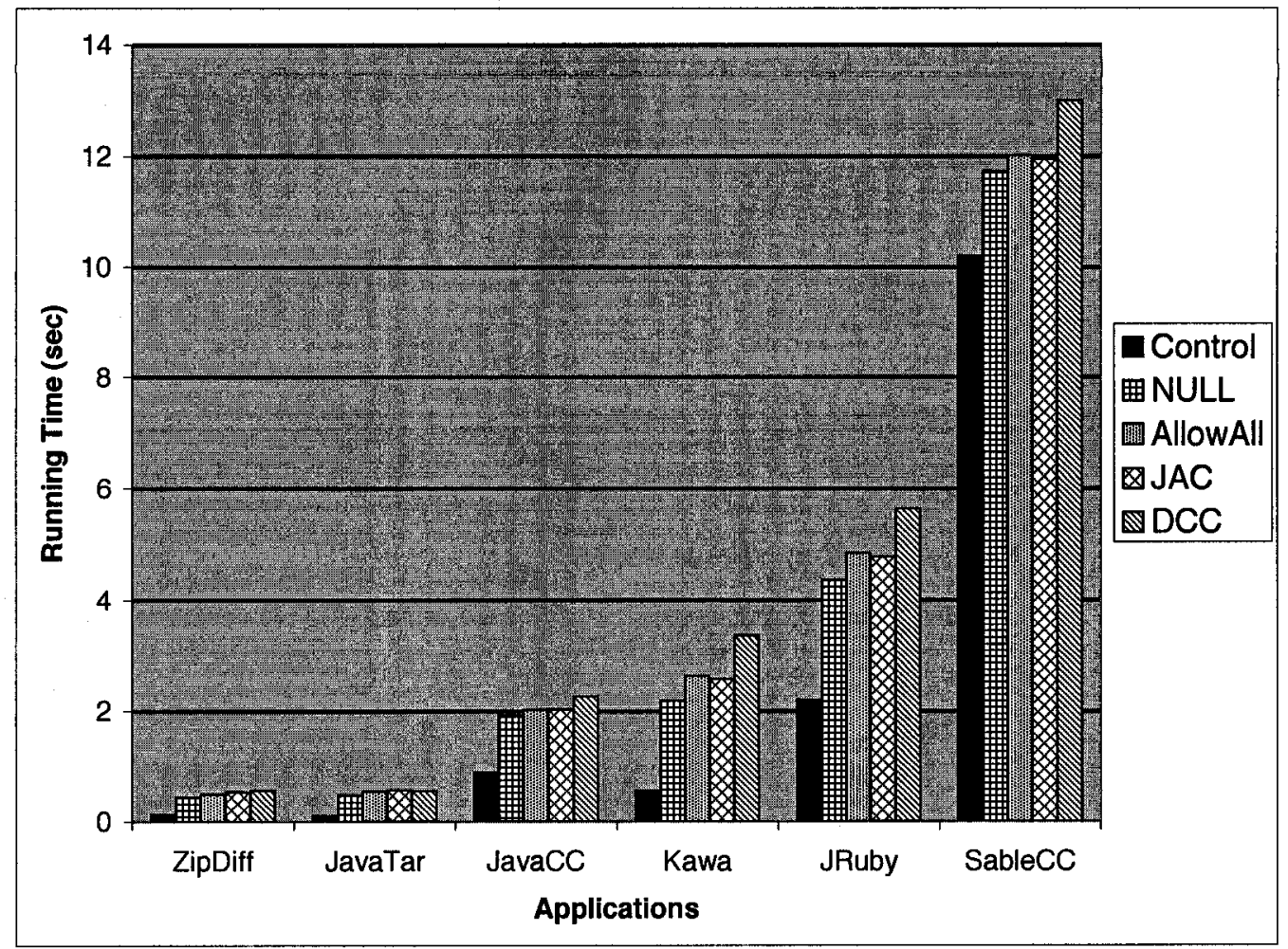

Figure 6.4: IsoMoD Overhead

able. By enforcing more complex policy clauses, such as $D C C$, the overhead increases. This particular result is expected as more conditional applicability checks must be made by the verification process. In our experiments with larger applications that execute longer, the overhead did not prove to be much of an encumbrance, since it never exceeded three seconds.

\subsection{Composition of overhead}

Analysis of the above results revealed two major components comprising IsoMoD's overhead:

- Mirror Maintenance: the process involved in constructing mirror objects described in a classfile. This process involved loading the classfile, constructing mirror objects for each method and field in the type interface. Supertypes un- 


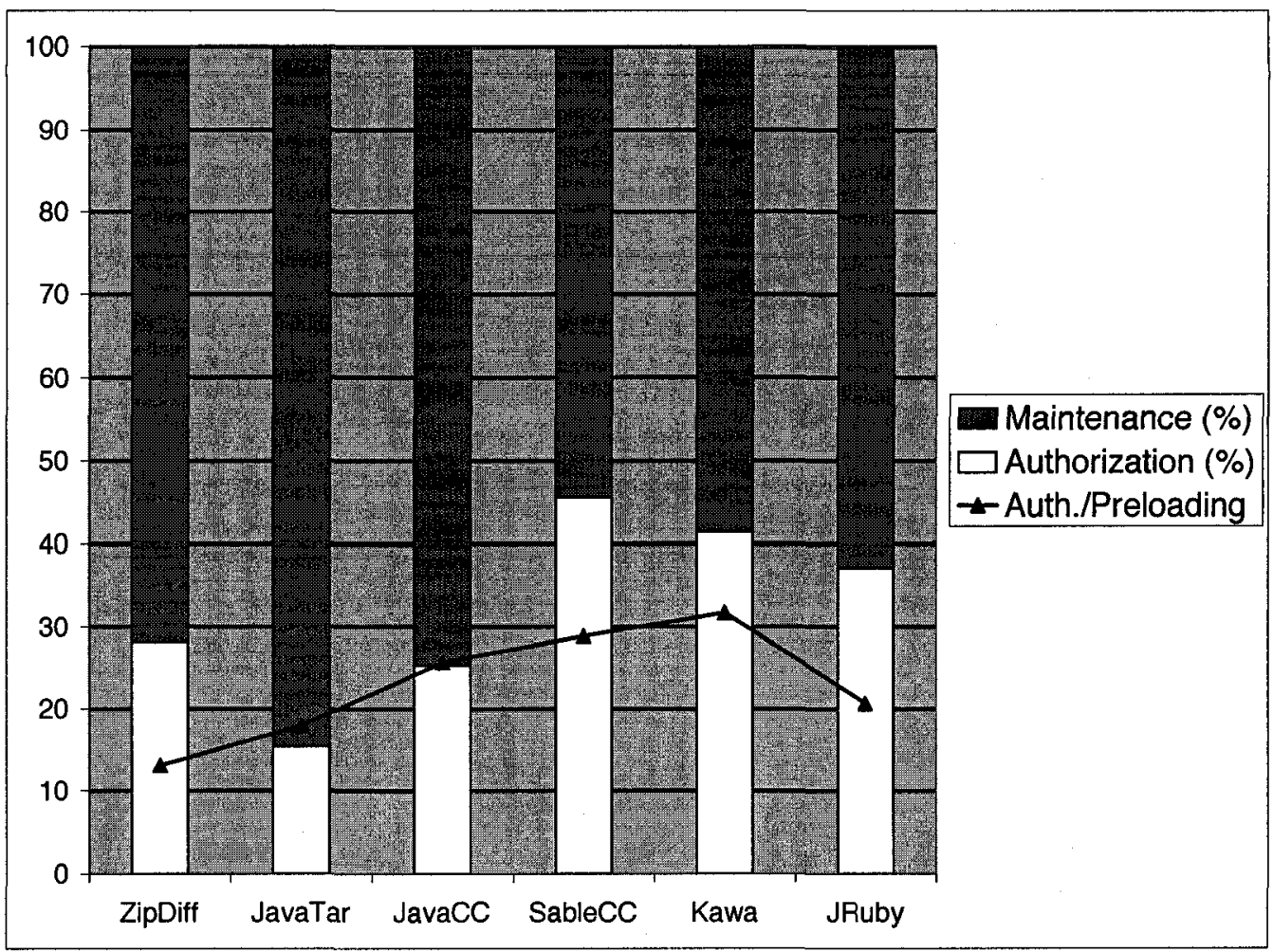

Figure 6.5: Composition of Overhead

dergo a similar construction. External accesses described in the classfile are examined, and corresponding access mirror objects are constructed and recorded. The overhead of this process can be easily measured by subtracting the running time of the Control configuration from the running time of the NULL configuration. This is because the NULL configuration contains no policy clauses. With no policy clauses, access mirrors are not subjected to applicability evaluation. The access mirrors are iterated through, but since no IsoMoD policy clauses exist, all accesses will be immediately allowed. Since no access evaluation occurred, IsoMoD only performed Mirror Maintenance.

- Access Authorization: this is the process of authorizing access mirrors against IsoMoD policy clauses. Each access collected for a given classfile must be authorized against relevant policy clauses. These clauses can involve applicability 
of conditions of various complexity. Complex conditions require more time to evaluate when authorizing access mirrors. This process can be measured by taking the difference in the running time between the $N U L L$ configuration and a non-trivial configuration, say, $D C C$. The NULL configuration takes into account the time spent on Mirror Maintenance. $D C C$ includes complex access mirror applicability checks as well as Mirror Maintenance. By removing the time spent on the NULL configuration, we are left with the time spent on $A c$ cess Authorization.

Figure 6.5 depicts the percentage of time spent for each component of the overhead over the complete cycle of execution. It can be seen that most of the time is spent on Mirror Maintenance. On average, Mirror Maintenance required $68 \%$ of the time, while only $32 \%$ was needed for Access Authorization.

Also shown in Figure 6.5 is the ratio between the number of authorizations checked during a full execution and the number of preloadings performed. The latter figure is the total number of Stage-I, Stage-II, and Stage-III classfile preloads actually performed during a full execution.

This ratio relates the number of authorizations to the number of declared types that were preloaded. This ratio is associated with the composition of the overhead in a direct manner. As the number of access mirrors that are authorized increases, then so does the percentage of time spent on performing this process. This particular aspect can be seen directly when looking at the JavaTar configuration up to the SableCC configuration. The ratio is increasing, as does the percentage of processing time spent on Access Authorization. This direct correlation indicates that the number of accesses being authorized will have an impact on how much time is spent on Access Authorization. 


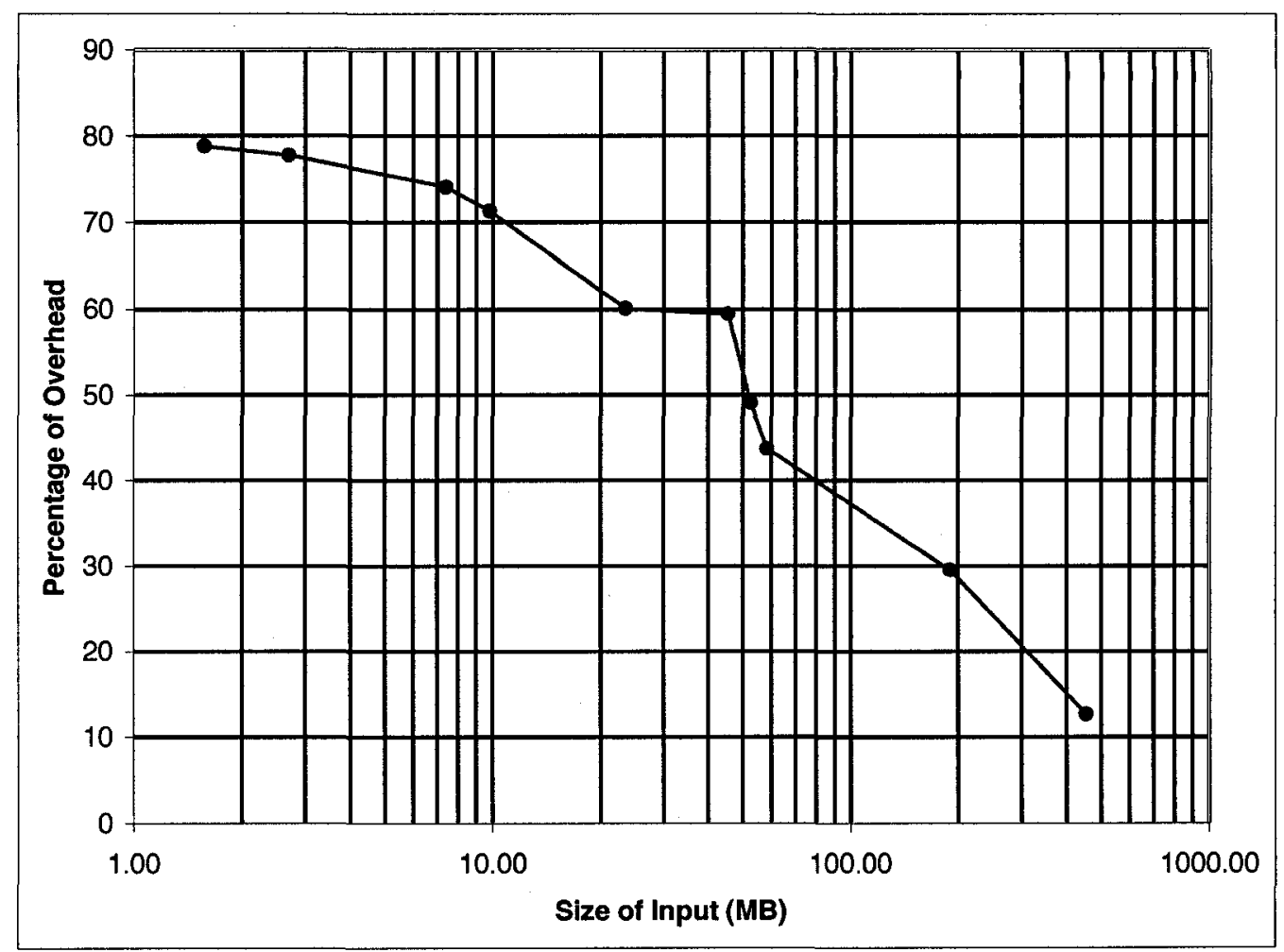

Figure 6.6: Penalty of Overhead

\subsection{Overhead distribution}

The goal of this experiment was to determine the percentage of the running time devoted to overhead during a long execution. Figure 6.6 describes Penalty of Overhead, as the percentage of running time given to IsoMoD during a complete execution. The size of the input is given in logarithmic scale because the input size varied from $24 \mathrm{~KB}$ up to $495 \mathrm{MB}$.

The JavaTar application creates an archive of files. There are two main reasons for choosing JavaTar in this experiment. First, the applications execution time could be varied, by varying the number of files to be archived. Second, since JavaTar was only operating on multiple sets of data, the number of mirrors constructed and the number of access mirrors recorded would be identical for all configurations. Third, JavaTar's performance during the first experiment (Section 6.3) was found to have 
the highest percentage of run-time devoted to overhead (although the magnitude of the overhead was small). Executing JavaTar through IsoMoD with the policy $D C C$ was found to have $81 \%$ of the execution time dedicated to overhead.

The graph in Figure 6.6 clearly demonstrates that as the input size was increased, the percentage of run-time spent on overhead decreased. The results of the experiment clearly indicated how a longer executing application reacted when executed through IsoMoD. Through each execution, the overhead incurred by IsoMoD was constant. This was because the policy used was never changed. The difference in execution times arises from varying the size of the input data.

The results of this experiment were as expected. Because the number of mirrors constructed and number of access mirrors authorized remained constant for each configuration, the amount of overhead incurred by IsoMoD remained constant and independent of the input data. This particular phenomenon of the overhead amortizing away would be even more visible in longer running applications, such as those found on servers. One could therefore enforce more complex policies on longer running application with a manageable overhead. The overhead incurred by enforcing these more complex policies would also be amortized away, as was demonstrated in this experiment.

\subsection{Summary}

Performance is one factor among many when determining if an approach to languagebased access control is viable. Since these experiments have demonstrated that the overhead incurred by ISOMOD is manageable and reasonable, they provide evidence of the usefulness of IsoMoD as a language-based access control mechanism. The IsOMOD class loader efficiently preloads and discharges accesses with small impact on the total execution time of an application. The most complex policy tested, $D C C$, 
incurred at most three seconds of overhead, which is reasonable. The authorization of accesses against policies was found to be minimal when compared to that of mirror maintenance. Because so little time is spent on authorization, more complex policies could be enforced with minor impact on the overhead. These results paved the way for exploring how IsoMoD behaved in a longer executing system. An application using the IsoMoD class loader that executed for a longer period of time had a lower percentage of time devoted to overhead. IsoMoD only incurred overhead during application startup, and this overhead amortizes away during a long run. 


\section{CONCLUSION}

This chapter concludes the thesis. Section 7.1 discusses related work. Section 7.2 summarizes the contributions made by this thesis, in the field of language-based access control. Section 7.3 discusses the limitations of ISOMOD and suggests future work.

\subsection{Related work}

Language-based software isolation has been achieved mostly by interposition-based mechanisms in the past. As surveyed in Chapter 2, early language-based systems such as Scheme 48 [28], Safe-Tcl [25] and SPIN [4] adopted namespace management as a primary protection mechanism. Two component mechanisms are involved. Firstly, dynamic linking dispatches monitoring code when system services are invoked (i.e., name resolution control). This is simply another form of Inlined Reference Monitors, and thus inheriting the performance issues of IRM. A Java incarnation of linkingbased interposition is described in [38]. Secondly, rudimentary name visibility control is employed to turn "on" or "off" certain names in a namespace. None of the policy clauses in Sections 4.2 and 4.3 can be enforced in this manner, because the visibility of names in these cases depends on complex relationships between the subjects and the objects. This thesis has thus demonstrated that name visibility control can in fact be much more expressive than is conventionally believed.

Module systems have been extensively studied in the past [18]. IsoMoD is a module system based in Java, and as such, some recent works relating to this area are discussed. JavaMod [2] is a module system for Java-like languages. The interaction between modularity and subtyping is carefully articulated. Another work by Bauer et al. [3] extends the Java package facility to obtain a module system that supports the decoration of import statements with linking obligations, which are in 
turn implemented as digital signatures. MJ [7] is a module system designed to control the complexity of configuration management in Java platforms. Liu and Smith [22] describe a module system that is based on a uniform framework for declaring explicit, bi-directional interfaces. Designed primarily for access control, IsoMoD is unique in two ways: (1) name visibility constraints can be imposed dynamically; (2) fine-grained name visibility constraints can be expressed in the IsoMoD policy language to precisely control what names are visible, to whom they are visible, and to what extent they are visible.

This work has been informed by the recent development of programmer-defined encapsulation policies $[29,30]$. Specifically, the designer of a class $A$ associates to $A$ a fixed number of access control policies, each presenting a different view of $A$. A client class $B$ then selects a policy through which $B$ interacts with $A$. Three points of comparison are worth noting. Firstly, because the client decides which policy to adopt, the scheme cannot be used for protection. Secondly, policies are formulated on a per-class basis, the universally quantified access control rules described in Section 4 cannot be expressed. Thirdly, IsoMoD defines a wider collection of access rights (e.g., "new", "catch", and "cast"), thereby differentiating finer levels of visibility.

\subsection{Contributions}

The scoping rules of a typical programming language are fixed. They prescribe the visibility of names in a given local namespace. Throughout this thesis, we entertain the idea of allowing an application to customize the scoping rules of a selected code region in which untrusted code resides. It has been demonstrated that this ability can serve as the cornerstone of an access control architecture for dynamically extensible systems.

The design of the aforementioned access control architecture is motivated by the 
engineering dilemma of interposition. As a popular language-based access control mechanism, interposition supports full execution monitoring by placing monitoring code at the entry point of security relevant services. Two variants of interposition have been proposed in the past: stack inspection and Inlined Reference Monitoring. While Java stack inspection can perform security checks fast and efficiently, the tight coupling of security to code is undesirable. Specifically, security checks are scattered throughout the source code of a system. A complete audit of the system is required when security requirements or software configurations evolve. The Inlined Reference Monitor approach separates policies from code, thereby allowing policies to evolve independently. While this approach is more desirable from a software engineering perspective, the degradation to performance is sometimes unacceptable.

While interposition can solve non-trivial problems, such as the confused deputy problem, in some systems, full execution monitoring is not necessary. IsoMoD is a lightweight alternative to interposition for language-based access control. IsoMoD achieves access control by exercising name visibility control over a local namespace. This work claims four contributions to the field of language-based access control:

1. an expressive, declarative policy language for specifying fine-grained name visibility constraints,

2. a protection mechanism for imposing name visibility policies over a namespace,

3. an efficient implementation strategy for realizing the above protection mechanism, and

4. an empirical study to provide evidence for the efficiency of the implementation strategy. 


\subsection{Limitations and future work}

Enforcement mechanisms that are based solely on static analysis, of which IsoMoD is an example, are provably less powerful than those that employ execution monitoring [16]. For example, policies in which the authorization decision is a function of invocation arguments or execution history are not enforceable by IsoMoD. This fact is demonstrated by IsoMoD only being able to capture a subset of the Java 2 permissions, for which runtime information is not needed for enforcement. The goal of this work was not to match the expressiveness of execution monitoring, but rather to find a lightweight alternative to interposition when full-fledged execution monitoring is not necessary.

A number of future directions are suggested by this work. Firstly, although IsoMOD provides a means for enforcing a given name visibility policy, it does not prescribe what policy to enforce in a given security context. IsOMOD could be extended into a full-fledged authorization system in the style of Java Authentication and Authorization Service (JAAS). Doing so would fill the aforementioned gap.

When a complex ISOMOD policy is given, it can be a non-trivial matter to understand if the policy is achieving what the author intends. Therefore, another direction is to construct a formal semantic model of IsOMOD. With such a semantic model, it would be possible to determine if a given safety property is enforced by an IsoMoD policy.

A third direction has to do with the pragmatics of using IsoMoD. This work provides an expressive policy language for defining scoping rules. With such expressiveness, it is possible for programmers to construct improper scoping rules, resulting in scoping disciplines that are incompatible with the typing disciplines of modern object-oriented programming languages. For example, how can one guarantee that a set of scoping rules honours the Principle of Subsumption [27]? Future work is necessary to address this challenge. 
A fourth direction would be to incorporate name resolution control. Name resolution control could be added when a class is preloaded. Certain methods would be "tagged" as sensitive. Whenever one of these sensitive methods is encountered, the name is resolved to a more secure method which will perform additional run-time security checks. This approach is similar to Safe-Tcl as discussed in Section 2.2.1.

A final direction is concerned with the automatic construction of IsoMoD policies. Policy engineering from scratch can be a tedious process. A remedy is to have a tool that will scan sample code units, and infer a policy that serves as the starting point of further policy engineering. 


\section{A AN IsoMod POLICY FOR DCC}

policy DCC

default allow

$/ /(\mathcal{D C C} 1)$

$\operatorname{method} B . N$

denies $\{$ invoke $\}$ to method $A . M$

unless (not $\operatorname{static}(N))$ or $\operatorname{trusts}(B, A)$

$/ /(\mathcal{D C C} 2)$

class $C$

denies $\{$ catch, cast, new $\}$ to method $A . M$

unless $\operatorname{trusts}(C, A)$

method B.N

denies $\{$ invoke $\}$ to method $A . M$

unless trusts(return-type $(N), A)$ or

(trusts $(A, B)$ and trusts $(B, A))$

field $B . F$

denies $\{$ get $\}$ to method A.M

unless trusts(field-type $(F), A)$ or

$(\operatorname{trusts}(A, B)$ and $\operatorname{trusts}(B, A))$

field $B . F$

denies $\{$ put $\}$ to method A.M

unless $\operatorname{trusts}(A$, field-type $(F))$ or

$(\operatorname{trusts}(A, B)$ and $\operatorname{trusts}(B, A))$ 
$/ /(\mathcal{D C C} 3)$

method B.N

denies $\{$ invoke $\}$ to method $A . M$

unless

(trusts $(A, B)$ and $\operatorname{trusts}(B, A))$ or

(for $C$ in parameter-types $(N)$ :

trusts $(C, B)$ or

$(\operatorname{trusts}(B, M)$ and $\operatorname{trusts}(C, M)))$

$/ /(\mathcal{D C C} 4)$

method $B . N$

denies $\{$ invoke $\}$ to method $A . M$

unless $\operatorname{trusts}(N, M)$

$/ /(\mathcal{D C C} 5)$

class $B$

denies $\{$ extend, implement $\}$ to class $A$ unless $\operatorname{trusts}(B, A)$

$/ /(\mathcal{D C C} 6)$

method $B . N$ 
denies $\{$ override $\}$ to method $A . M$

unless $\operatorname{trusts}(M, N)$

method $B . N$

denies $\{$ override $\}$ to method $A . M$

unless trusts(return-type $(N), B)$ or

(trusts $(A, B)$ and $\operatorname{trusts}(B, A))$

$\operatorname{method} B . N$

denies $\{$ override $\}$ to method $A . M$

unless

(trusts $(A, B)$ and $\operatorname{trusts}(B, A))$ or

(for $C$ in parameter-types $(N): \operatorname{trusts}(C, A)$ )

$/(\mathcal{D C C} 7)$

class $B$

denies $\{$ extend, implement $\}$ to class $A$

unless strongly-trusts $(B, A)$

$/ /(\mathcal{H M S} 1)$

class $C$

denies $\{$ extend $\}$ to class $\mathcal{E}$

unless

$\operatorname{domain}(\mathcal{E})$ implies

strongly-dominates $(\mathcal{E}$, org.aegis.dcc.Root)

$/ /(\mathcal{H M S} 2)$ 


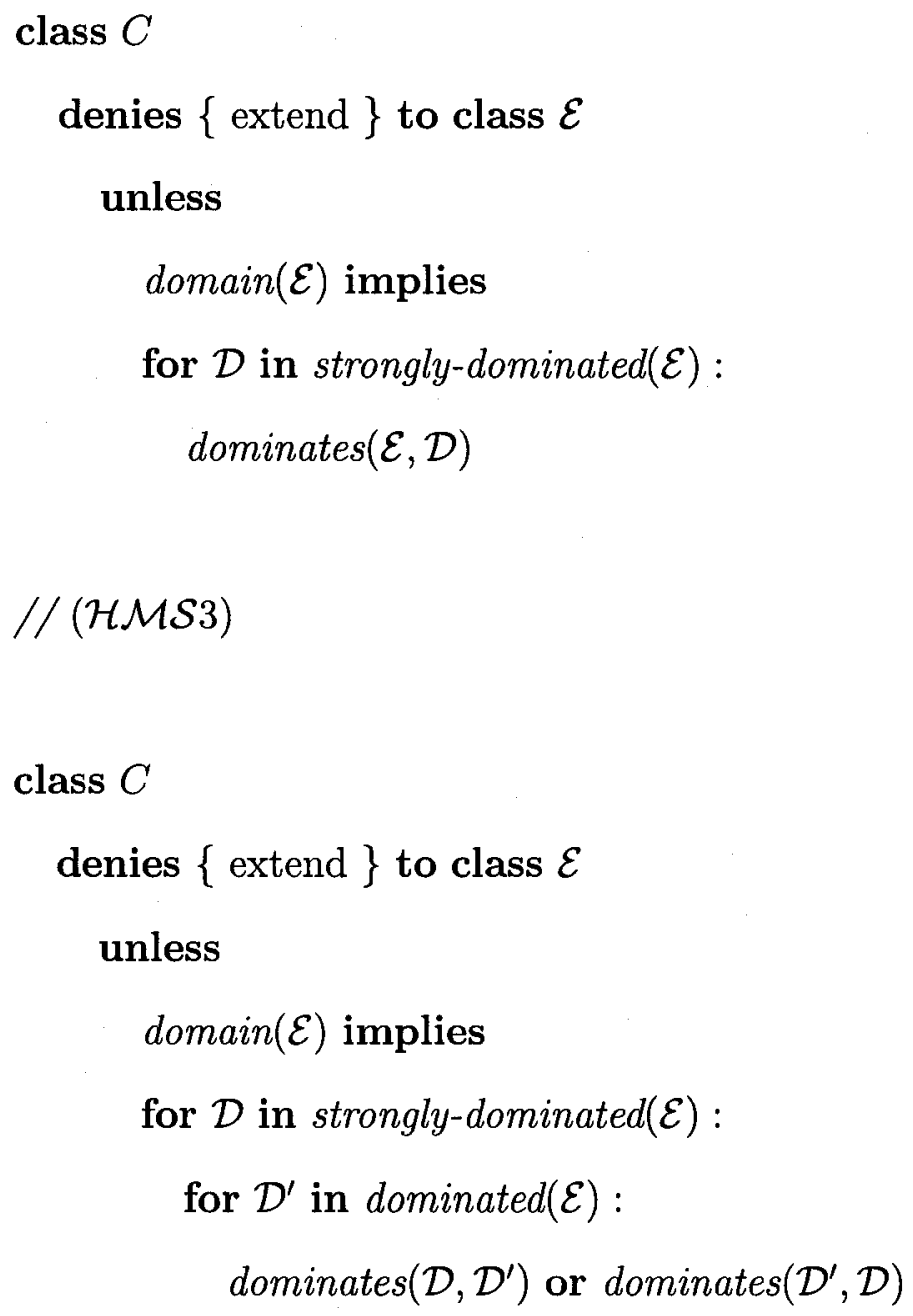




\section{B EXPERIMENTAL DATA}

\begin{tabular}{|l|c|c|}
\hline \multicolumn{3}{|c|}{ Program: JavaCC } \\
\hline Policy & $\begin{array}{c}\text { Standard Deviation } \\
\text { (in Seconds) }\end{array}$ & $\begin{array}{c}\text { Variance } \\
\text { (in Seconds) }\end{array}$ \\
\hline NULL & $3.92 * 10^{-3}$ & $1.50 * 10^{-5}$ \\
\hline AllowAll & $1.06 * 10^{-2}$ & $1.10 * 10^{-4}$ \\
\hline JAC & $6.50 * 10^{-3}$ & $4.18 * 10^{-5}$ \\
\hline DCC & $5.01 * 10^{-2}$ & $2.51 * 10^{-3}$ \\
\hline \hline \multicolumn{3}{|c|}{ Program: JavaTar } \\
\hline Policy & $\begin{array}{c}\text { Standard Deviation } \\
\text { (in Seconds) }\end{array}$ & $\begin{array}{c}\text { Variance } \\
\text { (in Seconds) }\end{array}$ \\
\hline NULL & $5.52 * 10^{-3}$ & $3.05 * 10^{-5}$ \\
\hline AllowAll & $4.52 * 10^{-3}$ & $2.05 * 10^{-5}$ \\
\hline JAC & $7.62 * 10^{-3}$ & $5.81 * 10^{-5}$ \\
\hline DCC & $4.04 * 10^{-3}$ & $1.63 * 10^{-5}$ \\
\hline \hline \multicolumn{3}{|c|}{ Program: JRuby } \\
\hline Policy & Standard Deviation & Variance \\
& (in Seconds) & (in Seconds) \\
\hline NULL & $1.23 * 10^{-2}$ & $1.52 * 10^{-4}$ \\
\hline AllowAll & $1.86 * 10^{-2}$ & $3.44 * 10^{-4}$ \\
\hline JAC & $1.57 * 10^{-2}$ & $2.5 * 10^{-4}$ \\
\hline DCC & $7.14 * 10^{-4}$ & $5.09 * 10^{-5}$ \\
\hline
\end{tabular}




\begin{tabular}{|l|c|c|}
\hline \multicolumn{3}{|c|}{ Program: Kawa } \\
\hline Policy & $\begin{array}{c}\text { Standard Deviation } \\
\text { (in Seconds) }\end{array}$ & $\begin{array}{c}\text { Variance } \\
\text { (in Seconds) }\end{array}$ \\
\hline NULL & $6.56 * 10^{-3}$ & $4.30 * 10^{-5}$ \\
\hline AllowAll & $3.36 * 10^{-3}$ & $1.13 * 10^{-5}$ \\
\hline JAC & $3.94 * 10^{-3}$ & $1.56 * 10^{-5}$ \\
\hline DCC & $1.65 * 10^{-1}$ & $2.73 * 10^{-2}$ \\
\hline \hline \multicolumn{3}{|c|}{ Program: SableCC } \\
\hline Policy & $\begin{array}{c}\text { Standard Deviation } \\
\text { (in Seconds) }\end{array}$ & $\begin{array}{c}\text { Variance } \\
\text { (in Seconds) }\end{array}$ \\
\hline NULL & $7.30 * 10^{-2}$ & $5.34 * 10^{-3}$ \\
\hline AllowAll & $4.50 * 10^{-2}$ & $2.02 * 10^{-3}$ \\
\hline JAC & $5.61 * 10^{-2}$ & $3.15 * 10^{-3}$ \\
\hline DCC & $9.13 * 10^{-2}$ & $8.34 * 10^{-3}$ \\
\hline \hline \multicolumn{3}{|c|}{ Program: ZipDiff } \\
\hline Policy & $\begin{array}{c}\text { Standard Deviation } \\
\text { (in Seconds) }\end{array}$ & Variance \\
& (in Seconds) \\
\hline NULL & $6.48 * 10^{-3}$ & $4.20 * 10^{-5}$ \\
\hline AllowAll & $7.55 * 10^{-3}$ & $5.70 * 10^{-5}$ \\
\hline JAC & $4.80 * 10^{-3}$ & $2.30 * 10^{-5}$ \\
\hline DCC & $3.89 * 10^{-3}$ & $1.51 * 10^{-5}$ \\
\hline
\end{tabular}




\section{List of References}

[1] M. Abadi and C. Fournet. Access control based on execution history. In Proceedings of the 10th Annual Network and Distributed System Security Symposium (NDSS '03), San Diego, California, USA, Feb. 2003.

[2] D. Ancona and E. Zucca. True modules for Java-like languages. In Proceedings of the 15th European Conference on Object-Oriented Programming (ECOOP '01), pages 354-380, Budapest, Hungary, June 2001.

[3] L. Bauer, A. W. Appel, and E. W. Felten. Mechanisms for secure modular programming in Java. Software - Practice \& Experience, 33(5):461-480, Apr. 2003.

[4] B. N. Bershad, S. Savage, P. Pardyak, E. G. Sirer, M. E. Fiuczynski, D. Becker, C. Chambers, and S. Eggers. Extensibility safety and performance in the SPIN operating system. In Proceedings of the 15th ACM Symposium on Operating Systems Principles (SOSP '95), pages 267-283, Copper Mountain, Colorado, USA, December 1995.

[5] G. Bracha and D. Ungar. Mirrors: Design principles for meta-level facilities of object-oriented programming languages. In Proceedings of the 19th ACM Conference on Object-Oriented Programming, Systems, Languages, and Applications (OOPSLA '04), pages 331-344, Vancouver, BC, Canada, Oct. 2004.

[6] D. D. Clark and D. R. Wilson. A comparison of commercial and military computer security policies. In Proceedings of the 1987 IEEE Symposium on Security and Privacy (S\&P '87), pages 184-194, May 1987.

[7] J. Corwin, D. F. Bacon, D. Grove, and C. Murthy. MJ: A rational module system for Java and its applications. In Proceedings of the 18th ACM Conference on 
Object-Oriented Programming, Systems, Languages, and Applications (OOPSLA '03), pages 241-254, Anaheim, CA, USA, Oct. 2003.

[8] J. B. Dennis and E. C. V. Horn. Programming semantics for multiprogrammed computations. Communications of the ACM, 9(3):143-155, March 1966.

[9] ECMA. Standard ECMA-335: Common Language Infrastructure (CLI), 2nd edition, Dec. 2002.

[10] D. Evans and A. Twyman. Flexible policy-directed code safety. In Proceedings of the 1999 IEEE Symposium on Security and Privacy (SßYP '99), pages 32-45, Oakland, California, USA, May 1999.

[11] P. W. L. Fong. Pluggable verification modules: An extensible protection mechanism for the JVM. In Proceedings of the 19th ACM Conference on ObjectOriented Programming, Systems, Languages, and Applications (OOPSLA '04), pages 404-418, Vancouver, BC, Canada, Oct. 2004.

[12] P. W. L. Fong. Discretionary capability confinement. In Proceedings of the 11th European Symposium on Research in Computer Security, Hamburg, Germany, September 2006. To appear. Also available as Technical Report CS-2006-03, Department of Computer Science, University of Regina.

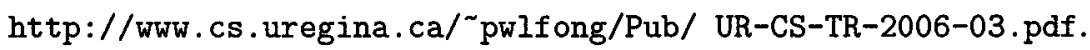

[13] P. W. L. Fong and R. D. Cameron. Proof linking: Modular verification of mobile programs in the presence of lazy, dynamic linking. ACM Transactions on Software Engineering and Methodology, 9(4):379-409, Oct. 2000.

[14] D. P. Friedman, M. Wand, and C. T. Haynes. Essentials of Programming Languages. MIT Press, 2nd edition, 2001. 
[15] L. Gong, G. Ellison, and M. Dageforde. Inside Java 2 Platform Security. Addison-Wesley, 2nd edition, 2003.

[16] K. W. Hamlen, G. Morrisett, and F. B. Schneider. Computability classes for enforcement mechanisms. ACM Transactions on Programming Languages and Systems, 28(1):175-205, Jan. 2006.

[17] N. Hardy. The confused deputy: or why capabilities might have been invented. Operating Systems Review, 22(4):36-38, Oct. 1988.

[18] R. Harper and B. C. Pierce. Design considerations for ML-style module systems. In B. C. Pierce, editor, Advanced Topics in Types and Programming Languages, chapter 8, pages 293-346. MIT Press, 2005.

[19] N. Li, Z. Bizri, and M. V. Tripunitara. On mutually-exclusive roles and separation of duty. In Proceedings of the 11th ACM Conference on Computer and Communications Security (CCS '04), pages 42-51, Washington DC, USA, October 2004 .

[20] S. Liang and G. Bracha. Dynamic class loading in the Java virtual machine. In Proceedings of the 13th ACM Conference on Object-Oriented Programming, Systems, Languages and Applications (OOPSLA '98), pages 36-44, Vancouver, BC, Canada, Oct. 1998.

[21] T. Lindholm and F. Yellin. The Java Virtual Machine Specification. Addison Wesley, 2nd edition, 1999.

[22] Y. D. Liu and S. F. Smith. Modules with interfaces for dynamic linking and communication. In Proceedings of the 18th European Conference on Object-Oriented Programming (ECOOP '04), Oslo, Norway, June 2004. 
[23] G. Morrisett, D. Walker, K. Crary, and N. Glew. From system F to typed assembly language. In ACM Transactions on Programming Languages and Systems (TOPLAS'99), volume 21, pages 527-568, 1999.

[24] G. C. Necula. Proof-carrying code. In Proceedings of the 24th ACM SIGPLANSIGACT Symposium on Principles of Programming Languages (POPL'97), pages 106-119, Paris, France, 1997.

[25] J. K. Ousterhout, J. Y. Levy, and B. B. Welch. The Safe-Tcl security model. In G. Vigna, editor, Mobile Agents and Security, volume 1419 of Lecture Notes in Computer Science. Springer-Verlag, 1998.

[26] D. L. Parnas. On the criteria to be used in decomposing systems into modules. Communications of the ACM, 15(12):1053-1058, December 1972.

[27] B. C. Pierce. Types and Programming Languages. MIT Press, 2002.

[28] J. A. Rees. A security kernel based on the lambda-calculus. A. I. Memo 1564, MIT, 1996.

[29] N. Schärli, A. Black, and S. Ducasse. Object-oriented encapsulation for dynamically typed languages. In Proceedings of the 19th ACM Conference on ObjectOriented Programming, Systems, Languages, and Applications (OOPSLA '04), pages 130-149, Vancouver, BC, Canada, Oct. 2004.

[30] N. Schärli, S. Ducasse, O. Nierstrasz, and R. Wuyts. Composable encapsulation policies. In Proceedings of the 18th European Conference on Object-Oriented Programming (ECOOP '04), Oslo, Norway, June 2004.

[31] F. B. Schneider. Enforceable security policies. ACM Transactions on Information and System Security, 3(1):30-50, Feb. 2000. 
[32] F. B. Schneider, G. Morrisett, and R. Harper. A language-based approach to security. In Informatics: 10 Years Back, 10 Years Ahead, volume 2000 of Lecture Notes in Computer Science, pages 86-101. Springer-Verlag, 2000.

[33] A. Snyder. Encapsulation and inheritance in object-oriented programming languages. In Conference Proceedings on Object-Oriented Programming Systems, Languages and Applications (OOPSLA '86), pages 38-45, Portland, Oregon, USA, September 1986.

[34] L. Snyder. Formal models of capability-based protection systems. IEEE Transactions on Computers, 30(3):172-181, Mar. 1981.

[35] Úlfar Erlingsson and F. B. Schneider. SASI enforcement of security policies: A retrospective. In Proceedings of the 1999 Workshop on New Security Paradigms (NSPW '99), pages 87-95, Caledon Hills, Ontario, Canada, September 1999.

[36] Úlfar Erlingsson and F. B. Schneider. IRM enforcement of Java stack inspection. In Proceedings of the 2000 IEEE Symposium on Security and Privacy (SEP '00), pages 246-255, Berkeley, CA, USA, May 2000.

[37] D. S. Wallach, A. W. Appel, and E. W. Felten. SAFKASI: A security mechanism for language-based systems. ACM Transactions on Software Engineering and Methodology, 9(4):341-378, Oct. 2000.

[38] D. S. Wallach, D. Balfanz, D. Dean, and E. W. Felten. Extensible security architecture for Java. In Proceedings of the 16th ACM Symposium on Operating Systems Principles (SOSP '97), pages 116-128, Saint Malo, France, Oct. 1997.

[39] I. Welch and R. J. Stroud. Using reflection as a mechanism for enforcing security policies on compiled code. Journal of Computer Security, 10(4):399-432, 2002. 
[40] A. W. A. with Jens Palsberg. Modern Compiler Implementation in Java. Cambridge University Press, 2nd edition, 2002. 